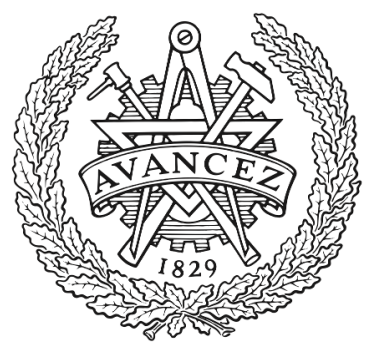

CHALMERS

UNIVERSITY OF TECHNOLOGY

\title{
Computational modeling of driver pre-crash brake response, with and without off-road glances: Parameterization using real-world crashes and
}

Downloaded from: https://research.chalmers.se, 2023-04-26 10:58 UTC

Citation for the original published paper (version of record):

Svärd, M., Markkula, G., Bärgman, J. et al (2021). Computational modeling of driver pre-crash brake response, with and without off-road glances:

Parameterization using real-world crashes and near-crashes. Accident Analysis and Prevention, 163. http://dx.doi.org/10.1016/j.aap.2021.106433

N.B. When citing this work, cite the original published paper. 


\title{
Computational modeling of driver pre-crash brake response, with and without off-road glances: Parameterization using real-world crashes and near-crashes
}

\author{
Malin Svärd ${ }^{\mathrm{a}, \mathrm{b},{ }^{*}, \text { Gustav Markkula }^{\mathrm{c}} \text {, Jonas Bärgman }}{ }^{\mathrm{b}}$, Trent Victor ${ }^{\mathrm{a}, \mathrm{b}}$ \\ ${ }^{a}$ Volvo Cars Safety Centre, 41878 Göteborg, Sweden \\ ${ }^{\mathrm{b}}$ Division of Vehicle Safety at the Department of Mechanics and Maritime Sciences, Chalmers University of Technology, 41296 Göteborg, Sweden \\ ${ }^{\mathrm{c}}$ Institute for Transport Studies, University of Leeds, LS2 9JT Leeds, United Kingdom
}

\section{A R T I C L E I N F O}

\section{Keywords:}

Driver behavior

Driver model

Glances

Brake response

Naturalistic data

PSO

\begin{abstract}
A B S T R A C T
When faced with an imminent collision threat, human vehicle drivers respond with braking in a manner which is stereotypical, yet modulated in complex ways by many factors, including the specific traffic situation and past driver eye movements. A computational model capturing these phenomena would have high applied value, for example in virtual vehicle safety testing methods, but existing models are either simplistic or not sufficiently validated. This paper extends an existing quantitative driver model for initiation and modulation of pre-crash brake response, to handle off-road glance behavior. The resulting models are fitted to time-series data from real-world naturalistic rear-end crashes and near-crashes. A stringent parameterization and model selection procedure is presented, based on particle swarm optimization and maximum likelihood estimation. A major contribution of this paper is the resulting first-ever fit of a computational model of human braking to real nearcrash and crash behavior data. The model selection results also permit novel conclusions regarding behavior and accident causation: Firstly, the results indicate that drivers have partial visual looming perception during offroad glances; that is, evidence for braking is collected, albeit at a slower pace, while the driver is looking away from the forward roadway. Secondly, the results suggest that an important causation factor in crashes without off-road glances may be a reduced responsiveness to visual looming, possibly associated with cognitive driver state (e.g., drowsiness or erroneous driver expectations). It is also demonstrated that a model parameterized on less-critical data, such as near-crashes, may also accurately reproduce driver behavior in highly critical situations, such as crashes.
\end{abstract}

\section{Introduction}

With an increasing range of advanced driver assistance systems (ADAS) becoming standard in new vehicles, there is a growing need of comprehensive assessment methods to evaluate the road safety of these systems. The use of virtual environments to evaluate driving safety is gaining popularity; consequently, validated, representative computational models of driver behavior in response to warnings and upcoming threats are becoming a necessity (see, for example, Bärgman, Boda, \& Dozza, 2017; Page et al., 2015). During the past decades, numerous models describing the driver's steering and/or braking control in various traffic situations have emerged (see reviews by Markkula, Benderius, Wolff, \& Wahde, 2012; Plöchl \& Edelmann, 2007). These models are useful for performing virtual simulations for road safety benefit analysis (Bärgman et al., 2017; Kusano and Gabler, 2012). However, most mathematical models of driver avoidance response are simplistic, based on a scenario-independent distribution of reaction times and predetermined intervention profiles, and typically assume that drivers will keep their eyes on the road (see, for example, the review of brake reaction times by Green, 2000). Since off-road glances are an inherent part of everyday driving, that assumption makes the models less realistic. Meanwhile, the National Highway Traffic Safety Administration (NHTSA) and other traffic authorities are imposing regulations restricting the placement of secondary tasks (Driver Focus-Telematics Working Group, 2006; Japan Automobile Manufacturers Association Inc., 2004; National Highway Traffic Safety Administration, 2016; The

\footnotetext{
* Corresponding author at: Volvo Cars Safety Centre, 41878 Göteborg, Sweden.

E-mail addresses: malin.svard@volvocars.com (M. Svärd), g.markkula@leeds.ac.uk (G. Markkula), jonas.bargman@chalmers.se (J. Bärgman), trent.victor@ volvocars.com (T. Victor).
} 


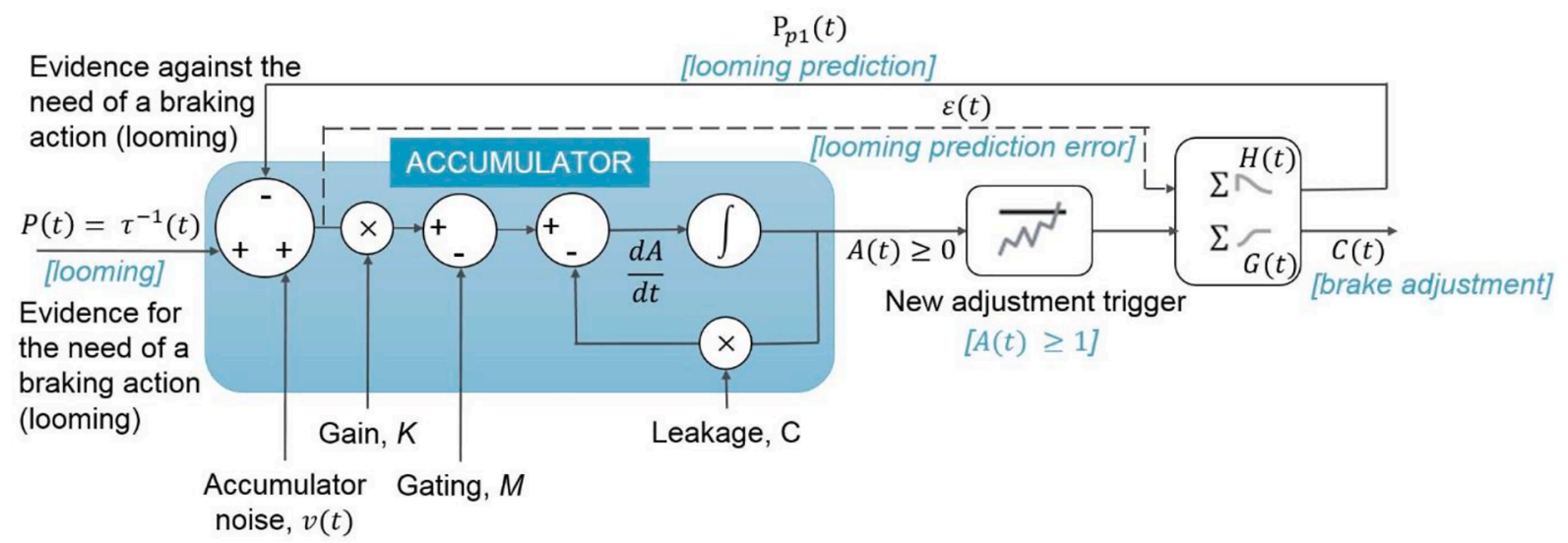

Fig. 1. Schematic representation of the model described by Svärd et al. (2017), extended with a leakage factor in the accumulator.

Commision of European Communities, 2008), as there are strong concerns that distractions from hand-held devices and in-vehicle displays will increase off-road glances and compromise safety. Furthermore, recent studies of naturalistic driving data from crashes and near-crashes suggest that the driver reaction is dependent on scenario kinematics (Markkula, 2014; Markkula et al., 2016), rather than being a fixed, scenario-independent, property of the driver (e.g., Kusano and Gabler, 2012).

To explain scenario-dependence, many authors have suggested that drivers decide on their avoidance actions based on perceptual cues such as visual looming, which is the optical size and expansion of a forward vehicle on the retina (Fajen, 2005; Flach et al., 2004; Lee, 1976; Markkula et al., 2016). Visual perception thresholds have also been used to determine detection of a forward threat in the modeling of driver control in near-crash situations (Kiefer et al., 2005). However, based on neuroscientific models of perceptual decision making and sensorimotor control, Markkula and colleagues (Markkula, 2014; Markkula et al., 2016) proposed that a driver's braking initiation is triggered, not exceeding a perceptual threshold, but rather by the accumulation of noisy perceptual evidence over time (best described by a nondeterministic model; Gold and Shadlen, 2007). Further, braking control also depends on the prediction of sensory consequences of primitive, open-loop, motor actions (Crapse and Sommer, 2008; Giszter, 2015; Markkula et al., 2018).

Based on the computational framework by Markkula and colleagues (Markkula, 2014; Markkula et al., 2018), a kinematics-dependent model quantifying pre-crash brake initiation and control has been proposed and applied to critical lead vehicle scenarios (Svärd et al., 2017). The model uses the accumulation of looming prediction error as the basis for the driver's braking response. Looming is quantified as in Eq. (1),

$\tau^{-1}=\frac{\dot{\theta}}{\theta}$

where $\theta$ is the optical size (width) of the lead vehicle on the driver's retina. Although Svärd et al. (2017) demonstrate that the model's brake initiation and ramp-up reproduce several qualitative trends observed in naturalistic crashes and near-crashes, the model has not yet been thoroughly parameterized and validated against such data.

Similar to most other perception based driver models, the model described by Svärd et al. (2017) is limited by the assumption that all perceptual input is disregarded during off-road glances. Studies have shown, however, that peripheral vision plays an important role in driving (Lamble et al., 1999; Land and Horwood, 1995; Lappi et al., 2017; Robertshaw and Wilkie, 2008; Summala et al., 1996; Wolfe et al., 2017). In fact, drivers are able to brake in response to an approaching lead vehicle, even when their gaze is constantly directed towards a secondary task, as demonstrated in the forced peripheral vision driving paradigm experiments performed by Summala, Lamble, \& Laakso (1998) and Lamble et al. (1999). However, since a relation between long duration off-road glances and increased crash risk has been demonstrated (Horrey and Wickens, 2007; Klauer et al., 2014; Victor et al., 2014), it would be beneficial to be able to model how, and to what extent, limited perceptual input influences driver brake response.

In this paper, the brake response model from Svärd et al. (2017) is extended to handle some accumulation of perceptual input during offroad glances. This is systematically done in two studies. The first study presents and compares four high-complexity models and is followed by a second study, reducing the complexity of the models presented in Study 1. A stringent parameterization of all model alternatives is accomplished using maximum likelihood estimation (MLE) on realworld naturalistic crashes and near-crashes, which are highly complex and more difficult to analyze than data collected in controlled studies (Carsten et al., 2013). Moreover, formal model selection is used to determine the benefit of the different mechanisms for handling driver off-road glances. All model alternatives are fitted to data from real-world crashes and near-crashes present in the second Strategic Highway Research Program Naturalistic Driving Study (SHRP2) (described in Victor et al., 2014).

\section{General method}

Svärd et al. (2017) describe a quantitative driver model for initiation and modulation of pre-crash brake response and apply it to critical lead vehicle scenarios. This paper describes the results from two consecutive studies, which extend that model by accounting for driver off-road glances and fitting the extended models to real-world naturalistic crashes and near-crashes. In Study 1, presented in Section 3, four highcomplexity model variants (that is, models with a high number of free parameters) are defined (see Section 3.1) and fitted on a crash dataset (see Section 3.2). Study 2, presented in Section 4, uses the findings from Study 1 to reduce the complexity of the models by setting a subset of the parameters to constant values. Four reduced-complexity model variants are introduced (see Section 4.1) and fitted on four (partially) overlapping datasets consisting of both crashes and near-crashes (see Section 4.2). Since the studies are closely coupled, the discussion of the results will not be individually presented, but is combined into a general discussion in Section 5.

This section gives a brief summary of the 2017-model by Svärd et al. (see Section 2.1; see the original publication for details) and the general data handling (see Section 2.2) and parameter fitting methods (see Section 2.3) used in the two studies. 


\subsection{Model description}

The model used in Svärd et al. (2017) is built on the computational framework developed by Markkula and colleagues (Markkula, 2014; Markkula et al., 2018). The model's brake initiation and modulation are based on four main principles of the framework:

- Braking is performed incrementally (i.e., in steps, in a series of "motor primitives").

- Brake initiation time is determined by the noisy accumulation of perceptual evidence for and against braking. The main evidence is the discrepancy between actual and predicted looming in terms of $\tau^{-1}(t)$, the looming prediction error $\varepsilon(t)$; see Eq. (1) for the definition of $\tau^{-1}(t)$.

- The amplitude of the brake adjustments is proportional to the looming prediction error at the time of brake adjustment initiation.

- After each incremental brake adjustment, the driver predicts how the looming will decrease as a result.

Once the accumulated evidence reaches a specific threshold, the driver issues a brake adjustment aimed at resolving the situation at hand. At each adjustment, the looming prediction error that is fed back to the accumulator is updated. This continues until either the critical situation is resolved, the maximum braking capacity of the vehicle is reached or a collision occurs. Fig. 1 illustrates the principles of the model.

As noted, in addition to the looming prediction error with a noise component, the accumulated evidence includes other factors that may influence the driver's brake response.

\subsubsection{Brake initiation}

The total accumulated evidence for the need of braking is denoted $A$ $(\mathrm{t})$. When this quantity reaches a specific threshold (set to 1 in this paper), a brake adjustment is initiated and the accumulated evidence is reset to a value $A_{r}$. Mathematically, evidence accumulation can be defined as in Eq. (2),

$\frac{d A(t)}{d t}=K \cdot \varepsilon(\mathrm{t})-\mathrm{M}-\mathrm{C} \cdot \mathrm{A}(\mathrm{t})+\mathrm{v}(\mathrm{t})$

where $K, M$, and $C$ are the free parameters gain, gating and leakage, respectively. The function $v(t)$ is the Gaussian zero-mean white noise at time $t$ with a standard deviation $\sigma \sqrt{\Delta t}$ for a model simulation time step of $\Delta t$.

The gain $K$ is a proportional constant determining the impact of the looming prediction error on the accumulated evidence (a higher $K$ will lead to more rapid accumulation); the gating $M$ effectively defines the minimum prediction error (or the minimum $\tau^{-1}(t)$, if the currently predicted looming is zero) required for evidence accumulation to commence. As described in (Markkula, 2014; Svärd et al., 2017), M may be thought of as the sum of all non-looming evidence for or against braking, which to some extent can be seen as a general expectancy of an upcoming need of braking. This is likely to include a wide range of situational factors, for example, general factors such as road type or traffic density, or discrete events: if the lead vehicle is far ahead and its brake lights activate, this might increase expectancy for braking, while if subsequently the lead vehicle turn indicators also activate (to signal that the lead vehicle will change lane), the expectancy might again decrease. Modelling these factors explicitly is beyond the scope of this paper, and $M$ can thus be thought of as representing an average level of expectancy across the modelled events.

In contrast with Svärd et al. (2017), we have also chosen to introduce a leakage term $C$ corresponding to the decay in the accumulated evidence over time, permitting some of the evidence to "leak out". This type of assumption is common in evidence accumulation models of decision making, and serves the purpose of truncating or "forgetting" outdated evidence (Usher \& McClelland, 2001; Nunes \& Gurney, 2016). Intuitively, if during car following $\tau^{-1}(t)$ briefly increases and then falls back to zero again, we wouldn't expect this episode to still be reflected in the value of $A(t)$ a minute or hour later.

\subsubsection{Brake modulation}

Each brake adjustment is determined by a piecewise linear function $G(t)$, which is scaled by the looming prediction error $\varepsilon(t)$ and a free brake gain parameter $k$. The total brake pedal signal $C(t)$ is the sum of all prior brake adjustments. At each brake adjustment, the future looming input is predicted to take the shape of a piecewise linear function $H(t)$, which is equal to one for a duration $\Delta T_{p 0}$, and then linearly decays to zero for a duration $\Delta T_{p 1}$. Both $\Delta T_{p 0}$ and $\Delta T_{p 1}$ are free model parameters. Based on the looming prediction error and the sum of all prior predictions, a total looming prediction signal $P_{p 1}(t)$ is calculated and fed back to the accumulator.

\subsection{Data}

To ensure that the model reflects real-world driver behavior, it was parameterized based on naturalistic data from real-world crashes and near-crashes collected in the SHRP2 naturalistic driving study (Transportation Research Board of the National Academy of Sciences, 2013). The dataset presented in Victor et al. (2014) was used (Transportation Research Board of the National Academy of Sciences, 2013), consisting of 46 crashes and 211 near-crashes categorized as rear-end (lead vehicle) situations (corresponding to scenarios 22-26 in the typology by Najm, Smith, \& Yanagisawa, 2007).

\subsubsection{Target scenario and dataset selection}

Data from real-world naturalistic crashes and near-crashes are highly variable, even when comparing events annotated as the same kind of scenario (e.g., rear-end). Hence, not all 46 crashes and 211 near-crashes were suitable for analysis in this paper. To facilitate the data selection, a target scenario that the driver model should be tailored to, was defined. The target scenario consists of rear-end situations on public roads (i.e., not parking lots or similar), without extreme driver states or visibility conditions. Moreover, road infrastructure should not be an obvious cause of lead vehicle braking expectancy. The main evasive maneuver performed by the driver should be braking (i.e., not steering), and it should be clear whether the pre-crash deceleration was the result of a driver intervention or the collision. Finally, all relevant signals should be available and of good enough quality. See Appendix A for more details regarding data selection.

The data selection process resulted in 13 crashes and 39 near-crashes (more near-crashes were available, but not necessary to create the final datasets). In the first study (the high-complexity models study), the 13 crashes were used for parameter fitting, while the fitting in the second study (the reduced-complexity models study) was performed on datasets which included progressively more and more near-crashes, with a decreasing level of severity (increasing minimum time-to-collision, TTC). Starting out with the crash dataset from the first study, an additional 39 near-crashes were appended in three increments of 13 nearcrashes each, resulting in the following four datasets used for parameter fitting in Study 2:

1. Dataset $13 c: 13$ crashes. (13 critical events.)

2. Dataset $13 c+13 n c: 13$ crashes and the 13 most severe near-crashes. (26 critical events.)

3. Dataset $13 c+26 n c$ : 13 crashes and the 26 most severe near-crashes. (39 critical events.)

4. Dataset $13 c+39 n c$ : 13 crashes and the 39 most severe near-crashes. (52 critical events.)

The critical events composing the datasets had a total of 49 distinct 
(a)

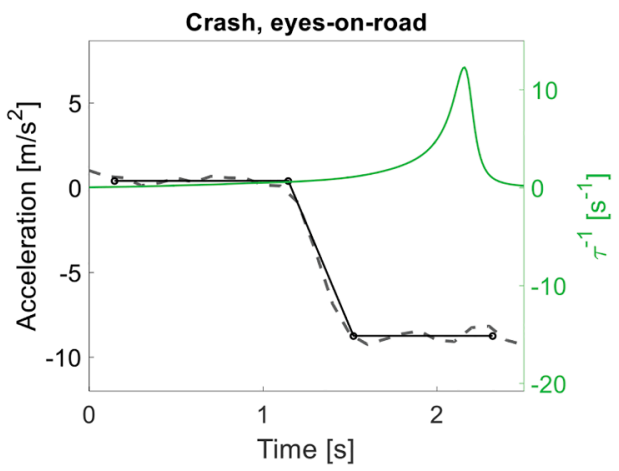

(c)

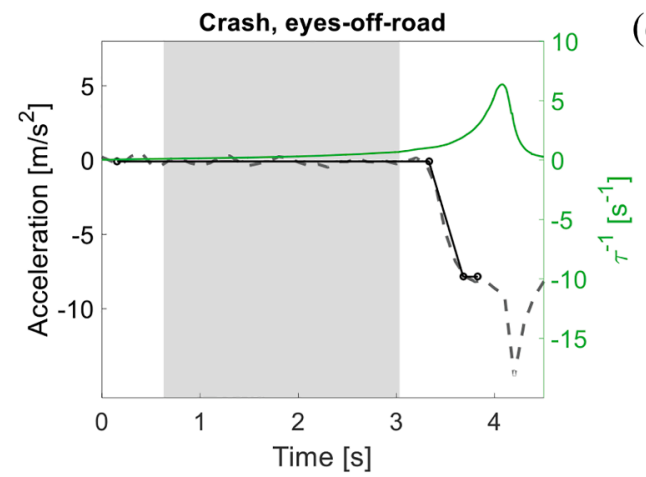

(b)

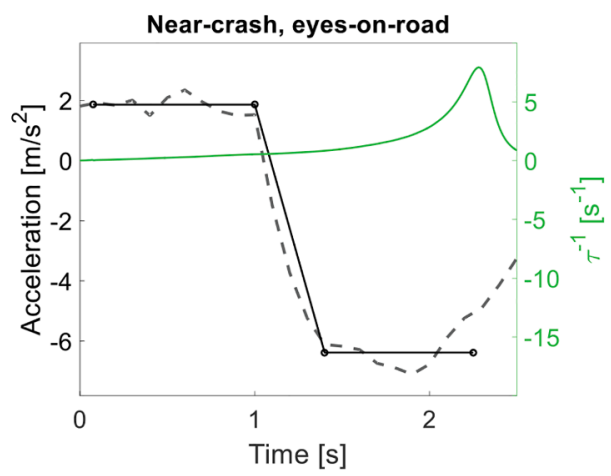

(d)

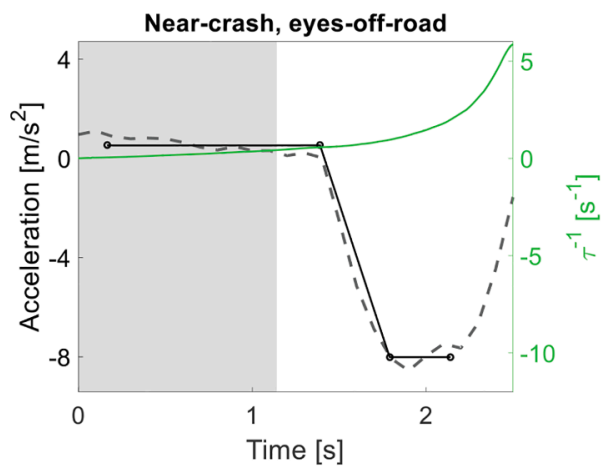

-- Acceleration (original) $\rightarrow$ Acceleration (fit) $-\tau^{-1}$

Fig. 2. Examples of looming profiles (green line) and piecewise linear model fitting (black line) of the acceleration signal (dashed gray line) for different types of events: (a) Crash without off-road glances, (b) near-crash without off-road glances, (c) crash with an off-road glance and (d) near-crash with an off-road glance. The gray areas illustrate timing and duration of the driver's glance off-road. (For interpretation of the references to color in this figure legend, the reader is referred to the web version of this article.)

drivers, with a relatively equal gender distribution (58 \% male and $42 \%$ female). The average driver age was approximately 30 years and the drivers had had their driving licenses for, on average, at least nine years. The driver demographics was relatively equal for all datasets, with the exception of dataset 13c (the crashes only dataset). Dataset 13c had a higher proportion of female drivers $(62 \%)$ and a lower average age (20-24 years), when compared to the full set of drivers.

\subsubsection{Data preparation}

The final selection of cases resulted in a total dataset of 52 rear-end events: 13 crashes and 39 near-crashes. All events were originally $20 \mathrm{~s}$ long, with the crash taking place at around $15 \mathrm{~s}$. Since the aim was to capture the driver's evasive braking behavior, not any potential speed reduction in advance of the actual critical event, only the last seconds before the crash/near-crash were of interest for the parameter fitting. The start of the event was defined to be the last moment in time before the point of collision (for crashes) or the minimum TTC (for nearcrashes), when the looming reached a minimum threshold value at the limit of human detection. The chosen threshold was $\dot{\theta}=0.0036 \mathrm{rad} / \mathrm{s}$, suggested by Morando, Victor, \& Dozza (2016) based on studies of visual perception thresholds by Summala, Lamble and Laakso (Lamble et al., 1999; Summala et al., 1998). Setting the detection threshold at $\dot{\theta}$ rather than at $\tau^{-1}$ lowers its sensitivity to environmental conditions (Morando et al., 2016), an advantage since our dataset consists of real-world naturalistic data.

The driver's evasive brake maneuver was removed from all cases, since it otherwise would have interfered with the situation's kinematics and hence influenced the brake response of the model if the model braked later than the human driver. As a result, the kinematics following the human driver's evasive braking was extrapolated from the previous kinematics in the event, assuming that the vehicle continued at a constant speed. The evasive maneuver removal process is described by Bärgman et al. (2017). Bärgman et al. (2017) and Victor et al. (2014) also describe in detail the process used to extract looming and reliable speed information from the original data. The used manual looming annotation method has been validated by Bärgman et al. (2013). Since the looming was computed using the derivative of a manually measured signal, noise could be a problem in cases with a high relative speed and a large distance to the vehicle ahead. The looming signal of the cases studied in this publication were manually examined to reduce the risk of issues related to noise.

One limitation of the available SHRP2 dataset is the lack of a brake pedal signal for most cases. Therefore, the brake initiation time and brake jerk were estimated by fitting the acceleration signal to a piecewise linear model, similar to what was done by Markkula et al. (2016). The model assumes a constant acceleration $a_{0}$ from the event start until a point in time $t_{B}$, which is defined as the brake initiation time. Starting at time $t_{B}$, the model linearly decays with a jerk $j_{B}$ until a final level of minimum acceleration $\mathrm{a}_{1}$ is reached. To correctly estimate the brake jerk in the reference cases (original recorded data) and in the model responses (simulations), the endpoint for the piecewise linear model fit was restricted to a point in time after the acceleration reached its minimum, but before it started to increase again. In crashes, the acceleration has a natural endpoint at the time of collision or at the start of evasive steering after the braking. On the other hand, in near-crashes finding the appropriate endpoint is more complex. Markkula et al.'s 2016 analysis of near-crashes used the point of minimum TTC $+0.5 \mathrm{~s}$ as the endpoint for the linear fitting since drivers generally maintained the minimum 
Table 1

Initialization ranges and boundaries for the free model parameters.

\begin{tabular}{lllllllll}
\hline Parameter & $M$ & $\sigma^{2}$ & $A_{r}$ & $k$ & $\Delta T_{p 0}$ & $\Delta T_{p 1}$ & $K$ & $C$ \\
\hline Initialization & {$[0$} & {$[0$} & {$[0$} & {$[0$} & {$[0$} & {$[0.05$} & {$[1$} & {$[0$} \\
$\quad$ range & $8]$ & $1]$ & $1]$ & $10]$ & $3.5]$ & $4.5]$ & $40]$ & $1]$ \\
\hline
\end{tabular}

acceleration for that long. This works well for the recorded data (reference events), but some model responses may not have reached their minimum acceleration by that point. Therefore, an additional condition was used: If a level of $95 \%$ of the minimum acceleration was not reached at minimum TTC $+0.5 \mathrm{~s}$, the endpoint would be set at the subsequent point in time when the acceleration reached $0.95 \%$ of the minimum acceleration, for the first time. See Fig. 2 for examples of the piecewise linear model fit for a set of crashes and near-crashes. Note that since the jerk signal was not computed directly from the acceleration signal, but estimated using the piecewise linear model which was continuous over the relevant interval (the brake maneuver), signal noise was not an issue.

\subsection{Parameter fitting}

Finding suitable parameter values for non-differentiable driver models with many free parameters (such as, in particular, the highcomplexity models in this paper) can be a complex task. Because of the high-dimensional search space, full grid-search, random search, and similar methods to find the optimal parameter values are inefficient and time-consuming. In addition, the optimization problem is required to be differentiable to use classic optimization procedures, such as gradient descent-based methods. Instead, a population based stochastic optimization method (PSO) was used to find a parameter set that maximizes the model fitness against the reference data. This metaheuristic method is suitable for searching very large solution spaces, though it cannot guarantee global optimality (Van Den Bergh and Engelbrecht, 2006; for details about PSO, see, e.g., Wahde, 2008, or Zhang et al., 2015). Here, brake model fitness is defined in a maximum likelihood sense-due to the stochastic nature of the model. The likelihood of a parameter set is estimated based on the results of Monte Carlo simulations.

\subsubsection{PSO implementation}

Initialization: The PSO was initialized with four particles per parameter (recommended population size for high PSO performance is usually 10-40 particles; Engelbrecht, 2007; Wahde, 2008), and each particle position was defined by randomly initialized parameter values (one value per parameter). See Table 1 for the initialization range for each parameter, which also define the feasible values for each parameter. Based on some initial tests, the ranges were selected to be narrow enough to minimize the parameter search space and keep the optimal values inside the feasible parameter ranges. The velocity of each particle was randomly initialized from a uniform distribution bounded on one side by the value of the particle position's upper limit and on the other by the negated value of the upper boundary, which is a simplification of the initialization procedure described for the standard PSO algorithm by Zhang et al. (2015).

Fitness calculation: In each iteration $k$ of the PSO algorithm, each reference event $i$ was simulated with 1000 Monte Carlo simulations for each potential parameter set $\mathbb{P}_{j, k}$, where $j$ is the particle number. Because of the noise term in the accumulator, each simulation resulted in a different model response. A piecewise linear function was fitted to the resulting acceleration profile from each simulation to determine the jerk level $j_{B, i}$ and brake initiation time $t_{B, i}$ for each event $i$ (as described in Section 2.2.2). The resulting $\left(t_{B, i}, j_{B, i}\right)$-values were then used to generate a two-dimensional probability distribution using Gaussian Kernel Density Estimation (KDE), in order to estimate the likelihood of the reference values $\left(t_{B, i}, j_{B, i}\right)$ of event $i$, given the current parameter set, denoted
$\ell\left(t_{B, i, r e f}, j_{B, i, r e f} \mid \mathbb{P}_{j, k}\right)$. In other words, the likelihood that the brake response from the actual event $i$ was generated by the driver model with parameter set $\mathbb{P}_{j, k}$ was estimated. If a simulation returned a nonresponse from the model (i.e., it did not perform evasive braking), the contribution to the KDE was set to 0 . Note that this means that the model was also fitted to the ratio of responses and non-responses in the dataset.

The Gaussian kernels used to generate the KDE were chosen so that the ratio of their standard deviations was approximately twice that of the ratio of the spread between $j_{B, \text { ref }}$ and $t_{B, r e f}$, see Eq. (3).

$\frac{\sigma_{j_{b}}}{\sigma_{t_{b}}}=2 \cdot \frac{\max _{i} j_{B, i, \text { ref }}-\min _{i} j_{B, i, r e f}}{\max _{i} t_{B, i, \text { ref }}-\min _{i} t_{B, i, r e f}}$,

This choice resulted in a kernel width of 3 in the $j_{B}$ dimension and 3/ 128 in the $t_{B}$ dimension. This scaling was necessary, not only due to their different orders of magnitudes, but also to prioritize a good fit of the brake onset timing over that of the jerk level during the optimization process. The reason for the prioritization was that brake initiation may be less dependent than the brake jerk on the chosen vehicle dynamics in the simulation, and, therefore, less sensitive to modeling errors (in, for example, the brake system model).

The total log-likelihood for the parameter set $\mathbb{P}_{j, k}$ was then calculated as the sum of the log-likelihoods for all $N$ reference events, according to Eq. (4),

$\log \mathscr{L}\left(\mathbb{P}_{\mathrm{j}, \mathrm{k}}\right)=\sum_{i=1}^{N} \log \ell\left(t_{B, i, r e f}, j_{B, i, r e f} \mid \mathbb{P}_{j, k}\right)$

To compensate for potential outliers that may contribute to an unnecessarily high value on the accumulator noise variance parameter $\sigma^{2}$, an additional outlier compensation term $p_{v}$ and a corresponding weighting factor $\rho$ were introduced. For each particle, the total loglikelihood was calculated according to Eq. (5):

$\log \mathscr{L}\left(\mathbb{P}_{\mathrm{j}, \mathrm{k}}\right)=\sum_{i=1}^{N} \log \left(\rho \bullet \ell\left(\mathrm{t}_{\mathrm{B}, \mathrm{i}, \mathrm{ref}}, \mathrm{j}_{\mathrm{B}, \mathrm{i}, \mathrm{ref}} \mid \mathbb{P}_{\mathrm{j}, \mathrm{k}}\right)+(1-\rho) \mathrm{p}_{\mathrm{v}}\right)$,

where $p_{\nu}=\frac{1}{t_{B, \max j_{B, \max }}}$. The latest possible brake initiation time and maximum brake jerk in the simulated model are denoted by and $t_{B, \max }$ and $j_{B, \max }$, respectively. Thus, in practice, the model fitness is a mix of a KDE distribution and a uniform distribution. The value of the weighting factor $\rho$ was chosen to minimize the variance $\sigma^{2}$ of the accumulator noise without noticeably reducing the log-likelihood of the optimal parameter sets in preliminary tests with different $\rho$ values (see Appendix B).

Position and velocity update: The velocity and position of each parameter in each particle were updated in each time step according to the method described by Shi and Eberhart (1998) and Wahde (2008); the cognitive and social components were both set to two. A linearly decaying inertia weight was used to gradually change the particle behavior from exploratory in the beginning to exploitative towards the end (its value ranged from 1.4 in the first iteration to 0.4 in the last). In addition, the particle velocity was restricted to maintain coherence among the particles.

\section{Study 1: Fitting high-complexity models}

As with most other quantitative driver model concepts, the model in Svärd et al. (2017) assumes either that drivers keep their gaze on-road at all times or that there is no perceptual input influencing the driver behavior during off-road glances. There is, however, compelling evidence suggesting that drivers do make use of peripheral vision in driving. The aim of this study is to extend Svärd et al.'s model to accommodate drivers' glance behavior and parameterize it using complex naturalistic real-world crash and near-crash data. This study is the first step in investigating the effect of new concepts on the off-road glance behavior model's performance and parameter values. Clearly, introducing more free parameters to an already complex model will 
Table 2

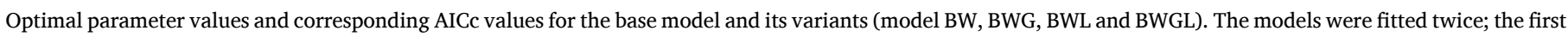

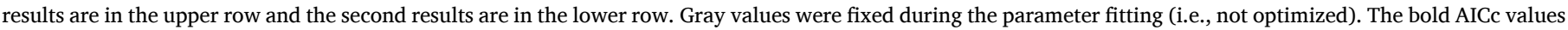
are the lowest in all compared models.

\begin{tabular}{|c|c|c|c|c|c|c|c|c|c|c|c|c|c|c|}
\hline Model & PSO run & AICc & $\log \mathscr{L}$ & $M$ & $\sigma^{2}$ & $A_{r}$ & $k$ & $\Delta T_{p 0}$ & $\Delta T_{p 1}$ & $K^{*}$ & $K_{o n}{ }^{*}$ & $K_{\text {off }}$ * & $w$ & $C$ \\
\hline \multirow[t]{2}{*}{ Base } & $1 \mathrm{st}$ & 153.69 & -58.64 & 5.77 & 0.96 & 0.96 & 1.34 & 1.12 & 3.23 & 32.88 & & & 0 & 0 \\
\hline & 2nd & 151.06 & -57.33 & 3.72 & 1.00 & 0.9 & 1.36 & 1.01 & 0.78 & 23.91 & & & 0 & 0 \\
\hline \multirow[t]{2}{*}{ BW } & $1 \mathrm{st}$ & 142.19 & -45.10 & 0,12 & 0.12 & 0.98 & 1.55 & 3.33 & 2.485 & 2.72 & & & 0.70 & 0 \\
\hline & 2nd & 145.06 & -46.53 & 2.27 & 0.73 & 0.98 & 1.60 & 0.57 & 1.93 & 7.88 & & & 0.67 & 0 \\
\hline \multirow[t]{2}{*}{ BWG } & $1 \mathrm{st}$ & 163.72 & -42.86 & 2.12 & 0.95 & 0.78 & 1.54 & 0.03 & 4.30 & & 7.38 & 20.33 & 0.16 & 0 \\
\hline & 2nd & 165.31 & -43.66 & 2.37 & 0.97 & 0.95 & 1.37 & 3.40 & 2.60 & & 7.29 & 20.14 & 0.19 & 0 \\
\hline \multirow[t]{2}{*}{ BWL } & $1 \mathrm{st}$ & 170.79 & -46.40 & 3.15 & 0.93 & 0.91 & 1.47 & 3.42 & 2.75 & 16.69 & & & 0.32 & 0.42 \\
\hline & 2nd & 169.60 & -45.80 & 9.17 & 0.97 & 0.34 & 1.77 & 0.9 & 3.88 & 2.68 & & & 0.80 & 0.87 \\
\hline \multirow[t]{2}{*}{ BWGL } & $1 \mathrm{st}$ & 225.76 & -47.88 & 6.86 & 0.63 & 0.07 & 2.35 & 1.25 & 4.05 & & 39.13 & 26.84 & 0.61 & 0.23 \\
\hline & 2nd & 224.53 & -47.26 & 6.18 & 0.45 & 0.45 & 2.35 & 2.67 & 1.60 & & 36.62 & 25.28 & 0.61 & 0.15 \\
\hline
\end{tabular}

*) The model variants have either one gain parameter K, or two separate gain parameters $K_{\text {on }}$ and $K_{\text {off }}$.

result in very high complexity, which may lead to poor model generalization. The results will be used in Study 2 (see Section 4), whose goal is reducing the model complexity without sacrificing performance.

\subsection{Model variants}

Experiments show that the driver's brake reaction in lead vehicle situations is delayed when the driver is looking off-road during the critical event (Lamble et al., 1999; Summala et al., 1998). Therefore, it can by hypothesized that drivers' behavior is less influenced by looming while they are looking away from the road (cf. Markkula, 2014), and introducing a scaling of the acquired evidence during off-road glances could lead to better model performance. This effect may be modelled in a parameter for partial looming perception during off-road glances (see below). Moreover, the mechanisms causing crashes when the drivers' gaze is directed off-road in the pre-crash phase may be different from the mechanisms causing crashes when the driver gaze remains on-road throughout. In eyes-on-road situations, for example, the cognitive driver state (e.g. drowsiness; see Ratcliff and Van Dongen, 2011) may influence the effective responsiveness to looming (Markkula et al., 2016). Cognitive driver state effects include effects due to (a) expectation inaccuracies, or (b) reduction in responsiveness due to sleep deprivation but exclude effects due to eye-closures or other loss of perceptual input. One way to capture these cognitive driver state differences in a driver model would be to let the looming responsiveness depend on driver state, by using different values of the gain parameter for eyes-on-road and eyes-off-road events (see below). In this study, the model described in Svärd et al., (2017) is extended using the concepts above, and the hypothesis that some of the already-accumulated evidence may decay over time.

The model by Svärd et al., (2017), henceforth called the base model, consists of seven free parameters. Based on this model, four highcomplexity (i.e., with a high number of free model parameters) model variants were defined by introducing different combinations of the following parameters:

1. An off-road glance looming weight parameter $w$, accounting for partial looming perception during off-road glances (for the parameter fitting initialized in the range $[0,1]$ ). This will permit brake responses to occur very quickly after an off-road glance, since the driver accumulates evidence also when directing their gaze off-road.

2. Different looming prediction error gains, $K_{o n}$ and $K_{\text {off }}$, depending on whether the gaze was on- or off-road during the event (for the parameter fitting initialized in the range $[1,40]$ ). This parameter aims to capture the differences in the underlying mechanisms for onand off-road glances in critical situations, by assuming that the cognitive driver states may influence the driver responsiveness to looming in on-road critical situations (leading to a delayed braking response).
3. Leakage $C$, as explained in Section 2.1 (for the parameter fitting initialized in the range $[0,1]$ ), which will help the model to not be overly sensitive to previous looming variations. Practically it is a decrease of the looming over time.

The following are descriptions of the created model variants:

Model BW (Base model extended with looming Weight): The base model was extended only with a looming weight parameter that accounts for the partial looming perception during off-road glances. This model variant has eight free parameters.

Model BWG (Base model extended with looming Weight and multiple Gains): Model BW was extended to include multiple looming prediction error gains, depending on whether the driver performs any off-road glance during the critical event. This model variant has nine free parameters.

Model BWL (Base model extended with looming Weight and Leakage): Model BW extended to include leakage. This model variant has nine free parameters.

Model BWGL (Base model extended with looming Weight, multiple Gains and Leakage): Model BWG extended to include leakage. This model variant has ten free parameters.

\subsection{Results}

The base model and its high-complexity variants (BW, BWG, BWL and BWGL) were fitted on dataset 13c, containing only crashes. All five were run with 250 PSO iterations, 1000 Monte Carlo simulations, and a $\rho$ value of 0.9 to compensate for outliers (see Appendix B for details about the $\rho$-value selection). The parameter fitting procedure was repeated once for each model to verify that the parameter values remained in the same range as in the first run. The performances of the model variants were compared using the Akaike Information Criterion with a correction for small sample sizes (AICc), which is a measure that balances goodness of fit and model complexity (Hurvich and Tsai, 1989; Sugiura, 1978). Within a set of candidate models, the preferred model is the one with the minimum AICc value. The Akaike Information Criterion (AIC) and Bayesian Information Criterion (BIC) were also calculated, and they essentially agreed with the AICc. (To reduce the complexity of this paper, the values of these criteria are not presented or further discussed.) The optimal parameter values and the corresponding AICc and log-likelihood values from both rounds of fitting are presented in Table 2.

As can be observed in Table 2, most parameter values were relatively consistent for the different parameter fittings. The only model variant outperforming the base model in terms of AICc was BW, extending the base model with a weighting parameter $w$ for partial looming perception during off-road glances. All model variants had a lower total loglikelihood value than the base model; due to their high complexity, 
Table 3

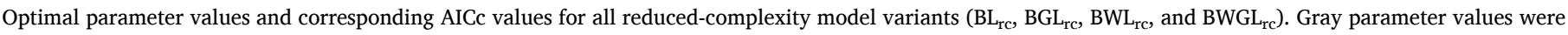
fixed (i.e., not optimized). Minimum AICc and maximum log-likelihood values among the compared model variants are marked in bold.

\begin{tabular}{|c|c|c|c|c|c|c|c|c|c|}
\hline Dataset & Model & AICc & $\log \mathscr{L}$ & $M$ & $\sigma^{2}$ & $K^{*}$ & $K_{o n} *$ & $K_{\text {off }}$ * & $w$ \\
\hline \multirow[t]{4}{*}{$13 c$} & $\mathrm{BL}_{\mathrm{rc}}$ & 123.80 & -57.57 & 2.28 & 0.99 & 14.43 & & & 0 \\
\hline & $\mathrm{BGL}_{\mathrm{rc}}$ & 109.85 & -48.43 & 0.01 & 0.15 & & 2.34 & 18.14 & 0 \\
\hline & $\mathrm{BWL}_{\mathrm{rc}}$ & 109.11 & -48.05 & 3.17 & 0.86 & 15.37 & & & 0.33 \\
\hline & BWGL $_{\mathrm{rc}}$ & 104.82 & -43.12 & 0.22 & 0.39 & & 3.01 & 18.63 & 0.04 \\
\hline \multirow[t]{4}{*}{$13 c+13 n c$} & $\mathrm{BL}_{\mathrm{rc}}$ & 263.23 & -127.28 & 0.87 & 0.80 & 8.61 & & & 0 \\
\hline & $\mathrm{BGL}_{\mathrm{rc}}$ & 259.61 & -123.30 & 0.09 & 0.48 & & 3.38 & 6.24 & 0 \\
\hline & $\mathrm{BWL}_{\mathrm{rc}}$ & 222.82 & -104.91 & 0.45 & 0.13 & 6.09 & & & 0.36 \\
\hline & BWGL $_{\mathrm{rc}}$ & 228.78 & -105.10 & 0.27 & 0.12 & & 6.79 & 6.52 & 0.31 \\
\hline \multirow[t]{4}{*}{$13 c+26 n c$} & $\mathrm{BL}_{\mathrm{rc}}$ & 390.40 & -190.87 & 0.01 & 0.54 & 4.64 & & & 0 \\
\hline & $\mathrm{BGL}_{\mathrm{rc}}$ & 386.75 & -186.87 & 0.02 & 0.53 & & 2.11 & 8.58 & 0 \\
\hline & $\mathrm{BWL}_{\mathrm{rc}}$ & 347.39 & -167.19 & 0.78 & 0.25 & 8.42 & & & 0.35 \\
\hline & BWGL $L_{r c}$ & 356.48 & -168.95 & 1.54 & 0.45 & & 10.63 & 10.72 & 0.35 \\
\hline \multirow[t]{4}{*}{$13 c+39 n c$} & $\mathrm{BL}_{\mathrm{rc}}$ & 569.46 & -280.39 & 0.00 & 0.25 & 5.50 & & & 0 \\
\hline & $\mathrm{BGL}_{\mathrm{rc}}$ & 568.03 & -277.51 & 0.17 & 0.53 & & 3.45 & 8.42 & 0 \\
\hline & $\mathrm{BWL}_{\mathrm{rc}}$ & 509.42 & -248.21 & 0.35 & 0.18 & 6.26 & & & 0.31 \\
\hline & BWGL $_{\mathrm{rc}}$ & 514.71 & -248.07 & 0.32 & 0.13 & & 5.97 & 5.5 & 0.38 \\
\hline
\end{tabular}

*) The model variants have either one gain parameter K, or two separate gain parameters $K_{\text {on }}$ and $K_{\text {off }}$.

models BWG, BWL, and BWGL were penalized in the AICc calculation to reduce the risk of poor model generalization.

\section{Study 2: Fitting reduced-complexity models}

A review of the model fitting results for the high-complexity models analyzed in Study 1 reveals that some of the parameters take on very similar values in most of the model variants. This consistency indicates that these parameters may not vary much between drivers and/or situations and could thus be set to constant values, improving generalizability without compromising model performance markedly. A further motivation for reducing model complexity this way is that, because of the high number of parameters, only one of the model variants in Study 1 performed better than the base model (in terms of AICc).

The aim of this study is to reduce the complexity of the models from Study 1 while keeping their ability to account for off-road glances and then to fit the reduced-complexity model variants to both crash and near-crash data (as described in Section 2.2.1). Further analyses were also carried out to study how the model's parameter values vary between combinations of datasets and modeling alternatives, and to identify specific critical events where one or more models align poorly with the observed human behavior.

\subsection{Model variants}

Parameters from Study 1 whose values were relatively unchanged across model variants were set to constant values to reduce model complexity. As a first step, we decided to set the reset value $A_{r}$ to 1 (the value found in several of the optimal parameter sets from Study 1; see Table 2), so that the accumulation of evidence was not reset at the time of brake intervention. To account for a realistic reduction in evidence accumulation over time, a leakage component was included in all model variants. The leakage parameter was fixed to $0.25 \mathrm{~s}$, in line with typical information decay timescales observed in primate cortex (Murray et al., 2014). Notably, this value is in the same range as the optimal values found when fitting models BWL and BWGL in Study 1 (ranging from 0.15 to 0.87 ). Three additional parameters were set to fixed values, based on the optimal values from the high-complexity model fitting: $k=1.3$, $\Delta T_{p 0}=1.5$ and $\Delta T_{p 1}=1.5$.

As a final step in the model complexity reduction, the off-road glance looming weight parameter was fixed at $w=0$ for two of the model variants ( $\mathrm{BL}_{\mathrm{rc}}$ and $\mathrm{BGL}_{\mathrm{rc}}$, defined below). This step is equivalent to removing the effect of partial looming perception during off-road glances, treating it the same way as in the base model (i.e., assuming no looming is accumulated while looking away).

To summarize, the parameter fixations resulted in the following four reduced-complexity model variants:

Model $B L_{r c}$ (Base model extended with Leakage, reduced-complexity): The base model extended with a fixed leakage parameter, $C=0.25$. This model variant has three free parameters.

Model BGL $L_{r c}$ (Base model extended with multiple Gains and Leakage, reduced-complexity): Model variant $\mathrm{BL}_{\mathrm{rc}}$ extended to include different looming prediction error gains depending on whether the driver performs any off-road glance during the critical event. This model variant has four free parameters.

Model BWL $L_{r c}$ (Base model extended with looming Weight and Leakage, reduced-complexity): Model variant $\mathrm{BL}_{\mathrm{rc}}$ extended with a looming weight parameter that accounts for partial looming perception during off-road glances. This model variant has four free parameters. Model BWGL $L_{r c}$ (Base model extended with looming Weight, multiple Gains and Leakage, reduced-complexity): Model variant $\mathrm{BWL}_{\mathrm{rc}}$ extended to include different looming prediction error gains depending on whether the driver performs any off-road glance during the critical event. This model variant has five free parameters.

\subsection{Results}

The reduced-complexity model variants $\left(\mathrm{BL}_{\mathrm{rc}}, \mathrm{BGL}_{\mathrm{rc}}, \mathrm{BWL}_{\mathrm{rc}}\right.$ and BWGL $L_{\mathrm{rc}}$ ) were parameterized on the four datasets described in Section 2.2.1. That is, each variant started out with the crash-only dataset and passed to datasets progressively including more near-crashes of lower criticality (longer minimum TTC). All PSO cycles were initially run with 250 iterations, then rerun with 500 or 750 iterations (depending on model complexity and dataset size) if convergence was not established. Details about the convergence analysis are presented in Appendix C. The optimal parameter values, the corresponding total log-likelihood, and the AICc value for each reduced-complexity model variant are presented in Table 3.

In Table 3, it can be observed that model $\mathrm{BWL}_{\mathrm{rc}}$ has the best performance in terms of AICc across all datasets, except dataset 13c (crashes only), where model $B W G L_{r c}$ is preferred. Overall, model variants $B W L_{r c}$ and $\mathrm{BWGL}_{\mathrm{rc}}$ have similar performances and parameter values. Models $\mathrm{BL}_{\mathrm{rc}}$ and $\mathrm{BGL}_{\mathrm{rc}}$ are also similar to each other, although their performances are somewhat poorer. Another important observation is that the gain parameters $K_{\text {on }}$ and $K_{\text {off }}$ take on values very close to each other for the most complex model variant with separate gains for eyes-on-road and eyes-off-road events (BWGL $L_{\mathrm{rc}}$ ). The parameter similarity is more 
(a)

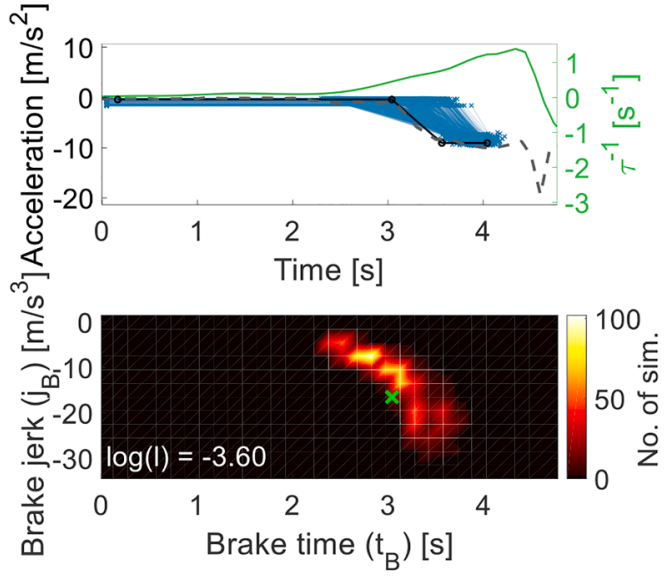

(c)

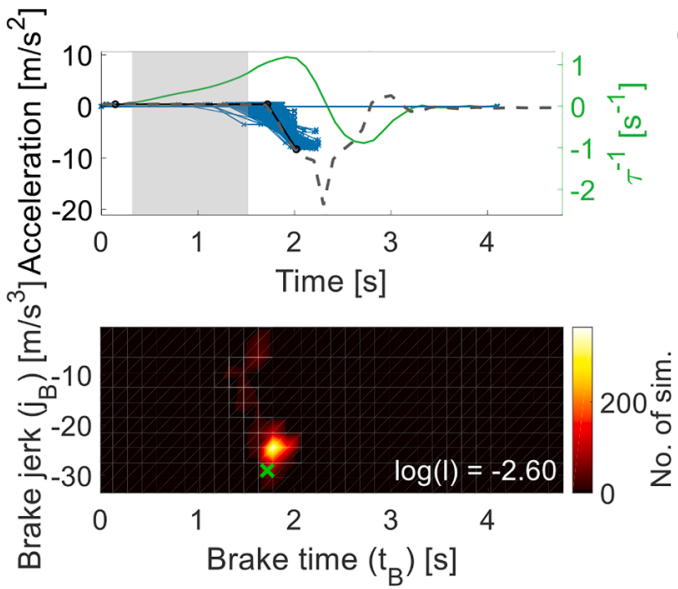

(e)

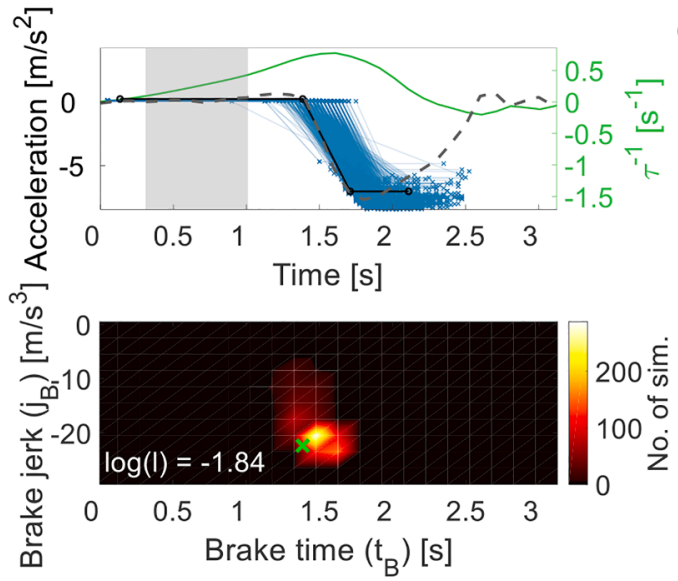

(b)

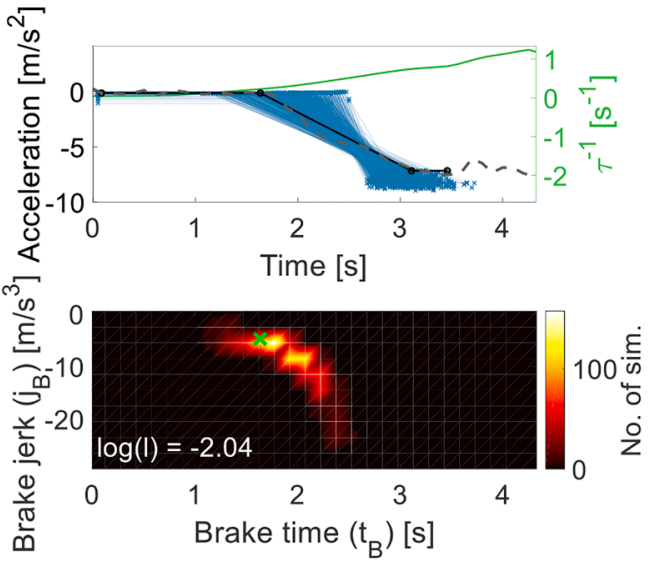

(d)

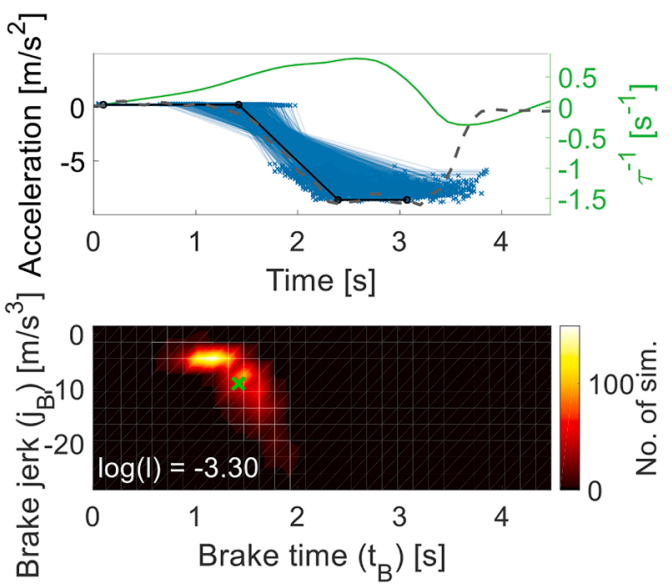

(f)

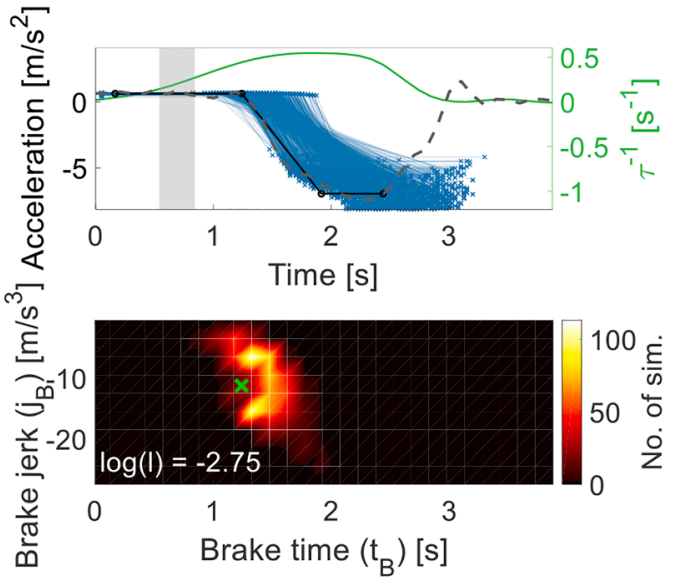

$-\tau^{-1}$ (original) ${ }^{--}$Acc. (original) $\rightarrow$ Acc. fit (original) $\rightarrow$ Acc. fit (model)

Fig. 3. Examples of events with good model performance. In each panel, the original and piecewise linear fitted acceleration from the reference event and the piecewise linear fitted acceleration from the model responses are shown in the upper graph, together with the looming curve. The lower graph shows the distribution of of $\left(t_{B}, j_{B}\right)$ values from all Monte Carlo simulations, as well as the $\left(t_{B, \text { ref }}, j_{B, \text { ref }}\right)$ value. Panels (a) \& (b): Crashes without an off-road glance; Panel (c): Crash with an off-road glance; Panel (d): Near-crash without an off-road glance; Panels (e) \& (f): Near-crashes with an off-road glance.

pronounced for the larger datasets.

The gating parameter $M$ converges to very small values on datasets $13 c+26 n c$ and $13 c+39 n c$, stopping at the boundary of the feasible set for model variants $\mathrm{BL}_{\mathrm{rc}}$ and $\mathrm{BGL}_{\mathrm{rc}}$. This may indicate that the optimal value is below the previously defined lower limit. Re-fitting with a lower boundary value, however, showed that even if the gating value goes below zero, the total model log-likelihood (and thus the AICc) does not change markedly. 
(a)

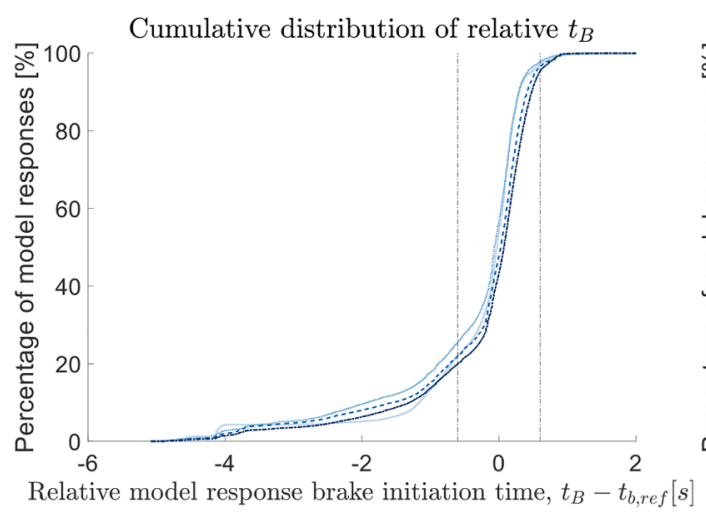

(b)

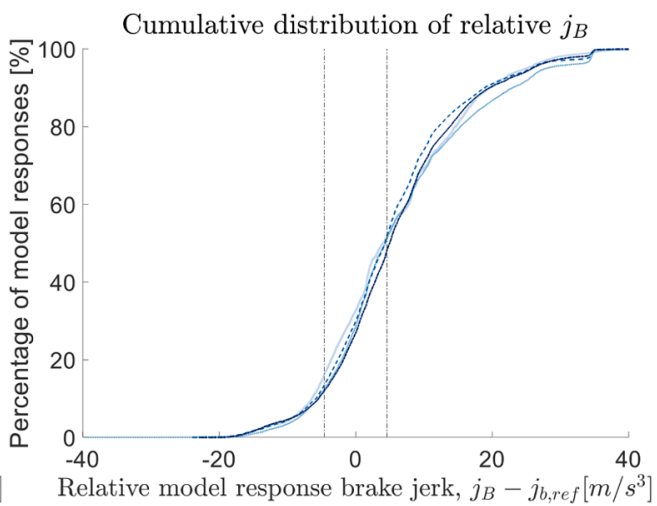

Dataset 13c $(n=13000) \cdots \cdot$ Dataset 13c+13nc $(n=26000)-\quad$ Dataset 13c+26nc $(n=39000)--$ Dataset 13c+39nc $(n=52000)$

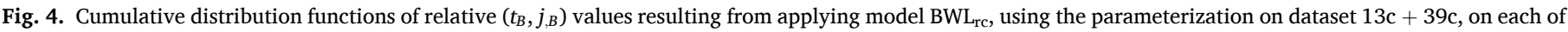

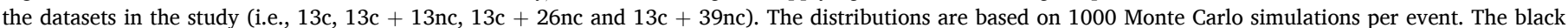
dashed lines represent the reference value $+/-0.5$ standard deviations. Panel (a): Distribution of $\left(t_{B}-t_{B, \text { ref }}\right)$ values; Panel (b): Distribution of $\left(j_{B}-j_{B, r e f}\right)$-values.
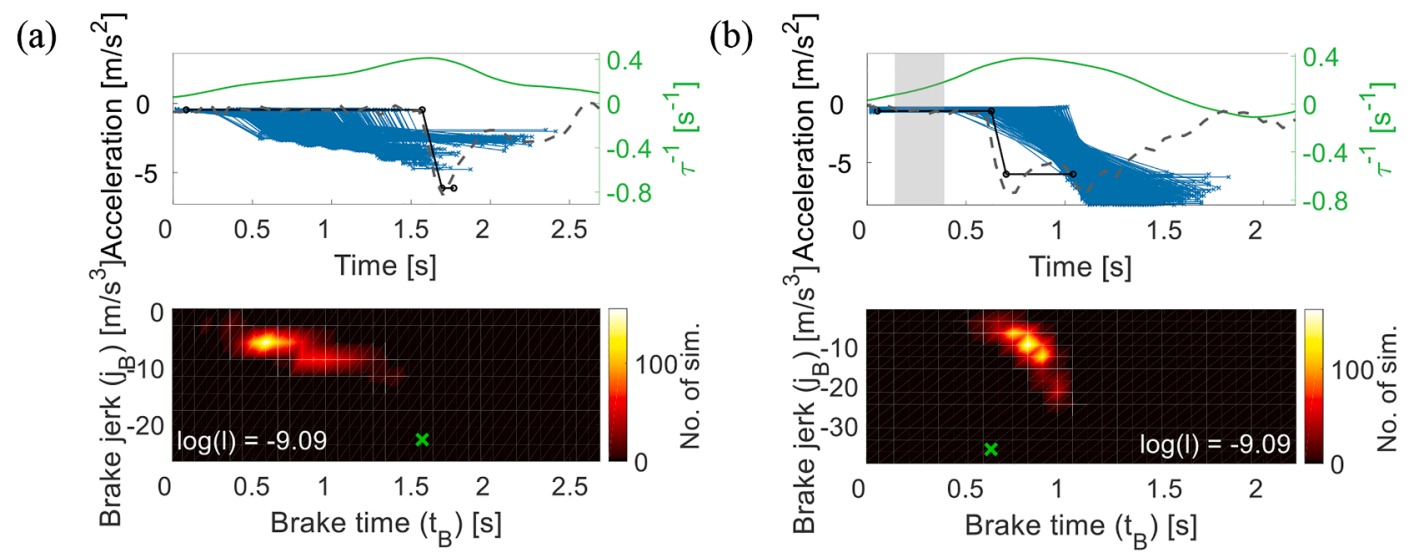

$$
-\tau^{-1} \text { (original) }-- \text { Acc. (original) } \rightarrow \text { Acc. fit (original) } \rightarrow \text { Acc. fit (model) }
$$

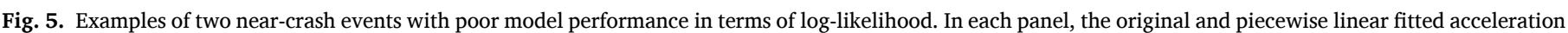

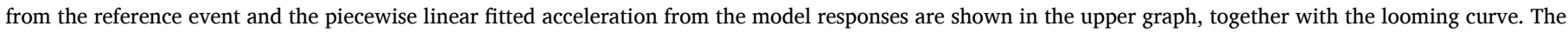

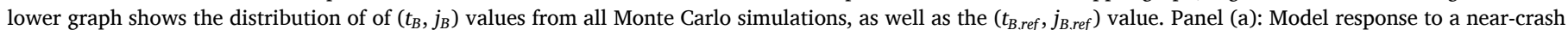
event without off-road glances, using model $\mathrm{BL}_{\mathrm{rc}}$; Panel (b): Model response to a near-crash event with an off-road glance, using model BWGL $\mathrm{r}_{\mathrm{rc}}$.

\subsubsection{Events with good overall model fit}

$59 \%$ of all model responses had individual log-likelihoods greater than -4.5 , corresponding to reasonably good fits (most of the Monte Carlo simulations had a brake initiation time and brake jerk that were close to the observed values in the reference event-approximately $50 \%$ of the simulations were within $+/-0.6 \mathrm{~s}$ for brake initiation time and $+/$ $4.6 \mathrm{~m} / \mathrm{s}^{3}$ for brake jerk). Fig. 3 shows the model response plots for some of these events, when applying BWL $\mathrm{L}_{\mathrm{rc}}$ (the variant with the lowest AICc) on three crashes and three near-crashes. The figure shows events in which the drivers were glancing off-road as well as events in which the drivers had their gaze on-road the whole time. For these illustrated events, the individual log-likelihood levels range from -3.6 to -2.0 for the crashes and from -3.3 to -1.8 for the near-crashes. Each panel is divided into two plots:

Upper plot: The uppermost plot shows the acceleration (dashed dark gray line) and looming ( $\tau^{-1}$; green line) of the reference event (for the looming signal, the evasive maneuver was first removed), as a function of time. The black solid line with circle-markers is the piecewise linear model fitted to the reference acceleration, which represents the acceleration behavior that the model is trying to reproduce. The blue lines with cross-markers depict the model responses from all Monte Carlo simulations, when the model (with optimal parameter settings) is applied to the reference event. Some plots also have a gray area behind the curves, illustrating that the driver is performing an off-road glance during that time interval.

Lower plot: The lower plot is a density plot of the distribution of $\left(t_{B}\right.$, $\left.j_{B}\right)$ values for all Monte Carlo simulations. The $\left(t_{B}, j_{B}\right)$ space is divided into bins of equal size $\left(0.2 \mathrm{~s}\right.$ in the $t_{B}$ dimension and $3 \mathrm{~m} / \mathrm{s}^{3}$ in the $j_{B}$ dimension). The number of Monte Carlo simulations in each bin is colorcoded according to the color bar to the right of the density plot. The reference value, $\left(t_{B, r e f}, j_{B, r e f}\right)$, is marked with a green cross.

\subsubsection{Effects of the progressive inclusion of less critical events}

The influence of the dataset was further studied by, for all datasets, comparing the distributions of $\left(t_{B}, j_{, B}\right)$ values relative to the $\left(t_{B, \text { ref }}, j_{B, \text { ref }}\right)$ values from the reference events- that is, the distributions of $\left(t_{B}-t_{B, \text { ref }}\right)$ 
(a)

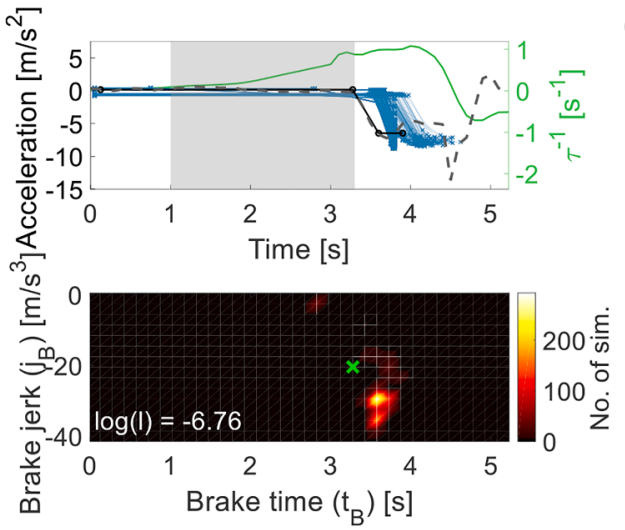

(b)

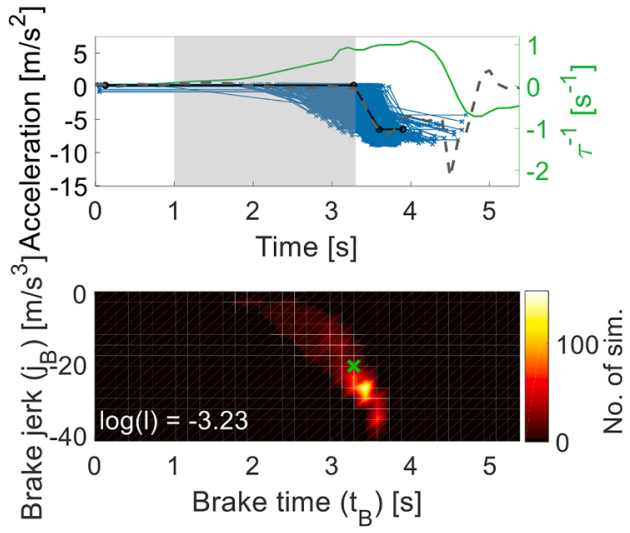

$\tau^{-1}$ (original) $)^{--}$Acc. (original) $\rightarrow$ Acc. fit (original) $\rightarrow$ Acc. fit (model)

Fig. 6. Examples of a crash event with different model performances, in terms of log-likelihood, depending on the applied model variant. In each panel, the original and piecewise linear fitted acceleration from the reference event and the piecewise linear fitted acceleration from the model responses are shown in the upper graph, together with the looming curve. The lower graph shows the distribution of of $\left(t_{B}, j_{B}\right)$ values from all Monte Carlo simulations, as well as the $\left(t_{B, \text { ref }}, j_{B, \text { ref }}\right)$ value. Panel (a): Model response using model $\mathrm{BL}_{\mathrm{rc}}$, showing a poor model fit; Panel (b): Model response using model BWGL $\mathrm{rc}_{\mathrm{rc}}$, illustrating a more accurate model fit.

and $\left(j_{B}-j_{B, r e f}\right)$. For this analysis, model BWL $L_{\mathrm{rc}}$, the model with the lowest AICc, was applied to all datasets (i.e., $13 \mathrm{c}, 13 \mathrm{c}+13 \mathrm{nc}, 13 \mathrm{c}+26 \mathrm{nc}$ and $13 c+39 n c)$, with the optimal parameter setting from fitting to dataset $13 c+39 n c$ (i.e., the largest dataset).

Including fewer severe near-crashes in consecutive datasets resulted in relatively minor changes in the optimal parameter values for each model variant, in particular for the larger datasets (see Table 3). Panel (a) in Fig. 4 shows the cumulative distribution function (CDF) of the relative $t_{B}$ values; Panel (b) shows the corresponding $C D F$ for the relative $j_{B}$ values. It can be observed that the shape and position of CDFs are essentially constant across the datasets.

The quality of the model predictions can also be quantified by studying Fig. 4. For brake initiation, $74 \%$ of the simulated data from all reduced-complexity model variants falls within $+/-0.6 \mathrm{~s}$ of the reference driver brake initiation time. This value corresponds to $+/-0.5$ standard deviations of the reference brake response times $\left(t_{B, \text { ref }}\right)$. The brake jerk prediction is somewhat poorer, with $37 \%$ falling within $+/-4.6 \mathrm{~m} / \mathrm{s}^{3}$ of the reference-corresponding to $+/-0.5$ standard deviations of the reference brake jerk $\left(j_{B, r e f}\right)$. A poorer estimate of brake jerk compared to brake initiation is to be expected, since a good $t_{B}$ fit was prioritized over the $j_{B}$ fit in the likelihood calculations: see Eq. (3).

\subsubsection{Model limitations for specific types of events}

The driver model variants are parameterized to perform well, in general, on a set of critical events with highly variable kinematics. Nonetheless, the variants might capture some driver behaviors better than others, because the model mechanics may be more suited for specific kinds of situations. To analyze how the individual critical events contributed to the model fit, the log-likelihood value for each event was studied for the complete set of parameter optimizations of reducedcomplexity models (i.e., 16 optimizations: models $\mathrm{BL}_{\mathrm{rc}}$, BGL $\mathrm{BL}_{\mathrm{rc}}, \mathrm{BWL}_{\mathrm{rc}}$ and BWGL ${ }_{\mathrm{rc}}$ on each of the datasets $13 \mathrm{c}, 13 \mathrm{c}+13 \mathrm{nc}, 13 \mathrm{c}+26 \mathrm{nc}$ and $13 c+39 n c)$. For most critical events, the log-likelihood values were similar across all datasets, but it was possible to distinguish between two main types of low-likelihood groups:

Events with low log-likelihood values $(<-8.5)$ across all model variants: Seven of the near-crash events, but none of the crashes, had a low performance for all model variants and datasets. In two of the events, the drivers had their gaze on-road, and in the five others the drivers had their gaze directed off-road at some point during the event. See Fig. 5 for two near-crash examples.

Events with low log-likelihood values for model variants $B L_{r c}$ and $B G L_{r c}$ but not for model variants $B W L_{r c}$ and $B W G L_{r c}$ : Four events had a low loglikelihood value for models $\mathrm{BL}_{\mathrm{rc}}$ and $B G \mathrm{~L}_{\mathrm{rc}}$, but not for models $B W \mathrm{~L}_{\mathrm{rc}}$ and $\mathrm{BWGL}_{\mathrm{rc}}$. The drivers were glancing off-road immediately prior to the critical situation, resulting in either a crash (two events) or a near-crash (two events). See Fig. 6 for a comparison of the distribution of $t_{B}$ and $j_{B}$ values for models $\mathrm{BL}_{r c}$ and $\mathrm{BWL}_{\mathrm{rc}}$ on one of the crash events.

\section{General discussion}

The two studies in this paper extend the non-deterministic driver model for brake onset and control presented by Svärd et al. (2017) to account for off-road glance behavior. The model performances of four model alternatives of high complexity were analyzed in Study 1; Study 2 reduced the model complexity and achieved models with good performance, fully parameterized on real-world naturalistic crash and nearcrash data, with fewer parameters than the original base model.

\subsection{Partial looming perception during off-road glances increases model performance}

The base model and the high-complexity model variants were parameterized only on the crash dataset (not the near-crash dataset), with the main aim of comparing the effects of including different aspects of the driver's glance behavior in the model. Model performance analyses (in terms of AICc) indicated that including partial looming perception during off-road glances was beneficial. Thus, it seems reasonable to conclude that drivers do collect information during offroad glances, presumably using their peripheral vision-as suggested in several previous studies (e.g., Lamble et al., 1999; Lappi, Rinkkala, \& Pekkanen, 2017; Heikki Summala, Nieminen, \& Punto, 1996; Wolfe, Dobres, Rosenholtz, \& Reimer, 2017; Wolfe, Sawyer, Kosovicheva, Reimer, \& Rosenholtz, 2019). In fact, there is conflicting evidence whether the retinal periphery is less able to detect collisions or react to looming. Studies by Li \& Laurent (2001) and Stoffregen \& Riccio (1990) indicate that (radial) looming perception is independent of retinal eccentricity. Further, Kim (2013) concluded that the peripheral retinal areas are actually more efficient than the center of the retina at judging impending collisions and controlling braking. However, the few studies on peripheral collision detection that have been performed in a vehicle setting, when the driver is not looking forward towards the roadway, showed delayed brake initiation timing with increased eccentricity (Burns et al., 2000; Lamble et al., 1999; Summala et al., 1998; Svärd 
et al., 2020). This finding indicates a sensitivity decrease for perceptual input processed by the peripheral vision system. This paper is further evidence of such sensitivity decrease. As far as we are aware, this paper is the first to demonstrate this phenomenon using real-world naturalistic crashes and near-crashes.

The benefits of including a partial looming perception parameter in the driver model could also be observed in the analysis of individual critical events with low log-likelihood values. Some of these events had a much higher log-likelihood when model variants including this parameter (i.e., BWL $L_{r c}$ and BWGL $L_{r c}$ ) were applied, compared to the model variants without it. The events were characterized by a late off-road glance, with the evasive braking maneuver occurring soon after the redirection of gaze. A high off-road gain $K_{\text {off }}$ (or $K$, if the variant had only one gain) could possibly compensate for a missing partial looming perception parameter, but at the price of poor model performance for other types of events.

\subsection{Cognitive driver state causes reduced looming responsiveness for crashes, but not for near-crashes}

The introduction of different gain factors for eyes-on-road and eyesoff-road events was motivated by the hypothesis that the mechanisms causing a situation to become critical depend on the cognitive driver state, as discussed by Victor et al. (2014). For example, the factors driving style (e.g., aggressive driving) and driver impairment (e.g., driver drowsiness) have been related to crash risk (Dingus et al., 2016). In fact, a mismatch between driver expectations and the upcoming situation may cause critical situations even in eyes-on-road events (Engström et al., 2018). However, in the eyes-off-road events, it is mainly the timing of the off-road-glance that causes the situation to become critical (Markkula et al., 2016; Victor et al., 2014). Thus, the cognitive driver state may cause a reduced responsiveness to looming input while looking on-road, which, in the models in this paper, can be reflected by a lower gain $K_{o n}$.

Here, the gain $K$ is used to make the distinction between eyes-onroad and eyes-off-road events in terms of, the potentially erroneous, driver expectations (which are different for on-road and off-road events), since it directly relates to the responsiveness to looming by scaling the looming prediction error. However, the gating $M$, which together with the gain $K$ determines the minimum predicted looming error required to initiate evidence accumulation, may also be seen as a general expectancy for the upcoming need of braking. In all model variants in this paper, the total driver expectancy is modeled by the gain and gating factors together.

In the current studies, model variants with different on- and off-road gains showed better performance on the crash dataset (in terms of both log-likelihood and AICc) than those with a single gain parameter-in line with the above hypothesis. Yet, this difference was not observed for the datasets including near-crashes. On these datasets (i.e., datasets $13 \mathrm{c}$ $+13 \mathrm{nc}, 13+26 \mathrm{nc}$ and $13 \mathrm{c}+39 \mathrm{nc}$ ), the model variants with two gains ( $K_{\text {on }}$ and $K_{\text {off }}$ ) performed only slightly better, in terms of AICc, than the corresponding variants with only a single gain parameter $(K)$. In addition, the gain values $K_{\text {on }}$ and $K_{\text {off }}$ for the most complex model variant $\left(\mathrm{BWGL}_{\mathrm{rc}}\right.$ ) turned out to be similar, in particular for the largest dataset. These observations indicate that there is no effect of cognitive driver state on perception responsiveness in near-crashes. One reason for this may be that the driver succeeds in resolving the critical situation, which indicates that drivers in near-crash scenarios may be more attentive (i.e., in another driver state) than drivers in crash scenarios.

\subsection{Parameterization on complex real-world naturalistic crashes and near-crashes results in reasonably good model fits}

A parameterization method based on PSO and MLE was proposed and applied to a number of non-deterministic driver models of different degrees of complexity. The method proved to be a useful tool for parameter fitting on highly complex naturalistic data. The model parameterizations resulted in reasonably good fits to the original data, in terms of brake initiation time and brake jerk (approximately $74 \%$ of the brake initiation times were within $+/-0.6 \mathrm{~s}$ and $37 \%$ of the estimated brake jerks were within $+/-4.6 \mathrm{~m} / \mathrm{s}^{3}$ from the human driver reference values). It is notable that we were able to achieve this level of performance on naturalistic real-world crash and near-crash data (as realworld data is inherently more noisy). Achieving the same results, using a full grid search method, for example, would not have been computationally feasible.

To decrease the risk of overfitting to the data, models with a low number of free parameters are preferable. For this reason the reducedcomplexity models were introduced in Study 2; they have fewer parameters (3-5) than the high-complexity models in Study 1 (8-10 parameters). As a result, the models were easier to analyze and resulted in a smaller search space for parameter fitting: it was easier to reach convergence and the parameter optimization required less computational capacity. However, if parameters that vary greatly for different drivers and/or situations are set to constant values, there is a risk of poorer model generalization (underfitting). Examples of poor model performance due to over- and underfitting are given in Awad \& Janson (1998), and the issues are also discussed by Lever, Krzywinski, \& Altman (2016). In the AICc analysis of the high- and reduced-complexity model variants on the crash dataset (dataset 13c), improved performance was observed for the reduced-complexity model variants. However, only the reduced-complexity model with the highest number of parameters had a better log-likelihood value than the high-complexity models. Together, these results indicate that the high-complexity models may overfit to the crash dataset, while the reduced-complexity models probably provide more opportunity for generalization when applied to new data (a desirable characteristic). The challenge is to find a driver model that is simple, yet close enough to the perceptual, cognitive, and motor mechanisms that are actually in play in critical situations. The chances of obtaining a model that generalizes well beyond the immediate dataset it was fitted to are maximized when the model captures biologicallyplausible mechanisms. This affords future model improvements to be made both while we discover things about the brain mechanisms and while we get more data.

\subsection{Models parameterized on less critical data are also able to reproduce driver behavior in more critical situations}

Including fewer severe near-crashes in the datasets resulted in relatively minor changes in the optimal parameter values for each individual model variant, in particular for the larger datasets. The number of crashes in naturalistic datasets are low compared to the number of nearcrashes, so it would be beneficial to be able to use less-critical data to parameterize driver models intended for highly critical situations (like the models in the current work) in order to draw conclusions about how driver behavior influences crash risk-for example in simulations for safety benefit estimations.

Analyzing data from the 100-car naturalistic driving study, Guo, Klauer, Hankey, \& Dingus (2010) showed that using near-crashes as surrogates for crashes provides a benefit when the amount of crash data is too low for the desired analysis. However, the authors point out that using near-crashes leads to a consistently underestimated crash risk. Later studies conclude that near-crashes are suitable as crash surrogates when studying collision risk, but it may be more challenging to use them to study crash severity (Tarko, 2018; see also the review by Zheng, Ismail, \& Meng, 2014). Thus, it may be expected that driver models fitted to near-crashes predict too-early driver interventions. Nonetheless, when studying model fit in terms of error distributions in the $t_{B}$ and $j_{B}$ dimensions, no obvious differences were found between the fit to crashes only and a mix of crashes and near-crashes, suggesting that a model parameterized on a less-critical dataset, at least in this case, 
successfully manages to reproduce the driver behavior even in more critical datasets to a reasonable extent. Our tentative conclusion from this data is that it is possible to use near-crashes for fitting to crashes, but that this would have to be confirmed in future studies.

However, due to the high variability in crash causation mechanisms and driver responses, the presented models are not suitable for analyzing driver behavior in all types of lead vehicle events. For a subset of the events in the analysis, all model variants performed poorly, indicating that there are still some mechanisms that are not captured by the models and/or parameterization method. Although somewhat speculative because of the small subset of events, the following observations can be made and are included to guide development of future models:

A major cause of poor performance was too-weak braking generated by the driver model (i.e., the human driver in the reference event braked harder). In the eyes-off-road events, the model braked later than the human driver, while it braked too early in the eyes-on-road events (with the exception of one event out of seven). Common factors for the eyesoff-road events were low looming and a long off-road glance. This caused the looming evidence to accumulate at a slower pace than in other events in the dataset, leading to a braking maneuver that was later and weaker than the reference maneuver performed by the human driver. In contrast, for the two eyes-on-road events, the initial looming was larger than for most other events in the dataset, causing the driver model to brake earlier than the human driver. Furthermore, it could be observed that in many of the events with too weak estimated brake jerk, the driver model responded to an early looming accumulation by issuing several individual brake adjustments spread out over time. Each individual brake adjustment could, however, have a strong jerk, matching that of the human driver. Nonetheless, the estimated mean brake jerk, from brake initiation until maximum brake power is reached (i.e., the estimated $j_{B}$ ), would be low because of the fitting to a piecewise linear model. The poor model performance for these events may thus be partly an effect of the parameterization method. A better performance might be achieved by calculating the brake jerk in several steps instead of one. That is, fitting a more advanced piecewise linear model to the acceleration signal. Further work to study these suggestions is recommended.

\subsection{Limitations and future work}

This work was based on real-world naturalistic crashes and nearcrashes from the SHRP2 database, which contained only a limited number of crash and near-crash events suitable for the specific analysis and model parameterization performed here. Thus, the parameter fittings in this paper were performed on datasets containing few crashes (n $=13$ ), where all crashes fulfilled the requirements of the target scenario (described in Section 2.2.1). The models fitted to the naturalistic data in this paper target specific crash mechanisms, and consequently, it should not be fitted to events that have different crash causation mechanisms (as the models are not designed to handle those). Examples of such mechanisms - thus reasons for event exclusion-are obvious driver drowsiness (i.e., easily spotted from video review) and driver expectancy due to infrastructure. In particular, fitting the models in this paper to critical events where the driver behavior is much influenced by expectancy would probably result in unreasonably high noise and gating values. This would result in a poor model fit on the crashes that matched the target scenario. To better understand the model limitations, more research is needed on the underlying factors and/or biological processes contributing to the parameter values in the current models (in particular, research on understanding the mechanisms related to the gating and leakage components).

A small dataset, particularly in combination with high-complexity models, may result in poor model generalization. To reduce this deficiency, near-crashes were appended to the dataset and lowercomplexity models were also created. However, due to limited data available and limited computational capacity, the largest dataset consisted of only 52 critical events. Accepting this limitation gave us the possibility of making several rounds of calculations where nine different model variants (inlcuding the base model) could be compared on four different datasets. The results indicate that including more near-crash events is likely to have little effect.

Moreover, the data in this study were collected in the United States and it is unclear whether the driver behavior could be generalized to other countries as well (in particular to developing countries with a different traffic pattern). In order to apply the model to, for example, safety benefit analysis, it should be re-parameterized on suitable data for the geocultural area of interest. It would also be reasonable to include age as a factor in the model, since younger drivers have been shown to have different glance patterns and different brake reactions than older drivers, possibly partially a result of this group of drivers more often being engaged in visual-manual secondary tasks (such as texting on the phone) at the time of an incident (Klauer et al., 2014; National Highway Traffic Safety Administration, 2020, 2012). This paper describes a method for parameterization, generalizable to any naturalistic dataset. The method could also be applied to parameterize other nondeterministic driver models which require the solution of a nondifferentiable optimization problem.

Parameterized and validated computational driver models of the type described in this paper (using real-world naturalistic driving data for parameter fitting) are an essential part of realistic virtual vehicle safety testing. Not only is there an increasing need of models aimed to evaluate the road safety of advanced driver assistance systems, such as forward collision warning systems (FCW) (see, e.g., Bärgman et al., 2017; Page et al., 2015), but computational driver behavior models may also be an important part of assessing the safety of automated driving systems (level 1-3) and driver comfort systems such as automatic cruise control (ACC) (Bianchi Piccinini et al., 2020). Consequently, models for other crash scenarios (e.g., intersection and run-off-road) should preferably also be fitted using naturalistic driving data.

\section{Conclusions}

This paper extends a driver model for brake onset and control to handle driver off-road glances and, for the first time, manages to fit a computational model to real-world naturalistic crash and near-crash data. A PSO- and MLE- based method was used to fit several model variants to real-world naturalistic crashes and near-crashes, and compare them using a structured model selection approach. The applied method is computationally efficient and permits parameter fitting of a non-deterministic model (i.e., including noise) with a large number of parameters. It was found that the best performing model variant is less complex than the original model, with only four free parameters: gain $K$, gating $M$, accumulator noise variance $\sigma^{2}$, and off-road glance looming weight $w$. The success of this reduced-complexity variant was probably due to the stringent model selection process that allowed model complexity to be reduced without compromising performance.

From the results in this paper, it was established that including partial looming perception during off-road glances, corresponding to $30-40 \%$ of the actual looming input, improved model fit and AICc. Thus it appears that drivers collect evidence for braking during off-road glances using the peripheral vision system, although they have less perceptual sensitivity than during on-road glances.

Moreover, we found evidence that some cognitive driver states (e.g., drowsiness or expectations that the situation will resolve itself without intervention) may cause a reduced responsiveness to looming. Thus 
driver state may be an important factor in determining of why crashes sometimes occur even when drivers keep their eyes on the road. This finding fills an important gap in the existing analyses of naturalistic crashes. However, reduced looming responsiveness does not seem to be a factor in near-crashes that occur while drivers have their eyes on the road.

Validated computational driver models is a critical part in virtual testing of vehicle safety systems (e.g. FCW), as well as in virtual assessment of comfort (e.g. ACC) and automated driving systems. The results from the reduced-complexity models in this paper, fitted to both crash and near-crash data, indicate that it is possible to reproduce driver behavior in critical situations using models parameterized on lesscritical events. However, a somewhat poorer performance was observed for specific kinds of events, in which the model brake response was weaker than that of the human driver. To overcome this limitation, a more advanced method for calculating the brake jerk could be used, and the model could be separately parameterized on a dataset containing more events of this kind.

\section{CRediT authorship contribution statement}

Malin Svärd: Conceptualization, Methodology, Software, Formal analysis, Investigation, Data curation, Writing - original draft, Writing review \& editing, Visualization, Project administration. Gustav Markkula: Conceptualization, Methodology, Writing - review \& editing. Jonas Bärgman: Methodology, Writing - review \& editing, Supervision. Trent Victor: Methodology, Writing - review \& editing, Supervision.

\section{Declaration of Competing Interest}

The authors declare the following financial interests/personal relationships which may be considered as potential competing interests: Malin Svärd and Trent Victor were, at the time this work was conducted, employed by Volvo Car Corporation, located in Gothenburg, Sweden. This study may impact how Volvo Car Corporation chooses to develop their products.

\section{Acknowledgement}

This research was funded by FFI Vinnova, Swedish governmental agency for innovation, as part of the project Quantitative Driver Behaviour Modelling for Active Safety Assessment Expansion (QUADRAE: nr. 2015-04863). Gustav Markkula received funding from the UK Engineering and Physical Sciences Research Council, grant EP/ S005056/1. The SHRP2 data used in this study has the identifier DOI SHRP2-DUL-16-172 and was made available to us by the Virginia Tech Transportation Institute (VTTI) under a Data License Agreement. The findings and conclusions of this paper are those of the authors and do not necessarily represent the views of VTTI, the Transportation Research Board (TRB), or the National Academies. The authors wish to thank Vignesh Krishnan at Volvo Cars Safety Centre for performing simulations and help setting up the analysis scripts used in this study; Daniel Irekvist for being a part of the method development, and specifically for his contributions in the PSO and MLE implementation; Fredrik Granum for fruitful discussions during the method development phase and for being a part of setting up the original the simulation environment used for this paper; and Kristina Mayberry for her language review.

\section{Appendix A. - Data selection}

\section{A.1 Selection of crashes}

The data in the original SHRP2 dataset were analyzed and reduced to only contain critical events matching the requirements of the target scenario, described in Section 2.2.1. The selection of crash events was done in two parts: (1) Signal based selection, and (2) video and description based selection, as follows:

Signal based selection: The signal based selection part ensured the availability of good quality data in terms of longitudinal kinematics and annotated looming, that is, all signals required by the driver model should exist and be complete (15 events did not fulfill this and were hence excluded). In addition, the following types of events were excluded:

- Events where it was not possible to separate the driver actions from the situation kinematics, for example, events where it was not clear whether the pre-crash deceleration was the result of driver intervention or the collision (three events excluded).

- Events where the difference between the piecewise linear acceleration fit and the original acceleration before the collision deviated too much (one event excluded).

- Events where the driver looked on-road for the entire event, but did not perform an evasive maneuver (one event excluded).

In total, 26 of the 46 rear-end crashes remained after the signal based selection.

Video and description based selection: In the video and description based selection process, the remaining 26 crashes were analyzed by looking at the forward view from the windshield mounted camera, and by reading the written description of the scenario made by the annotators. The following types of events were excluded:

- Events where the forward view through the windshield was not clear enough to expect a good quality looming annotation to be possible, for example as a result of a too blurry video image caused by night time rain (three events excluded).

- Events with noticeable evasive steering from the driver before the evasive brake maneuver (two events excluded).

- Events where the lead vehicle had an open trailer attached (one event excluded).

- Events where the driver was described as sleepy in the annotated event description (one event excluded).

- Events with extremely low speed, typically parking lot situations (two events excluded). 
- Events with possible issues with driver expectancy, for example expectations caused by a red light coming up in front (four events excluded).

In total, after the data selection, 13 good quality crashes (of the type targeted by the driver models in this paper) remained for the parameter fitting. A.2 Selection of near-crashes

The much higher amount of near-crashes than crashes in the original dataset warranted another method for determining inclusion or exclusion of events, than the rather time consuming procedure used for the crash dataset. First, all near-crashes with bad or missing (relevant) signals were discarded, as well as the near-crashes happening at very low speeds $(<20 \mathrm{~km} / \mathrm{h}$ at the moment when the driver was performing evasive braking). The remaining near-crashes were analyzed by visual inspection of the signals and forward video streams, excluding some cases with issues such as poor data quality, very bad visibility, events with evasive steering maneuvers, cut-in/out scenarios, and events where the lead vehicle was not a passenger car, in a similar manner to what was done for the crash dataset. All remaining near-crashes were ordered in terms of severity, where the severity of a near-crash was judged based on the minimum TTC during the event (this also corresponds to the highest looming). After this exclusion process, the 39 most severe good-quality near-crashes were selected for the parameter fitting (limited to this amount to keep a good balance between the number of crashes and near-crashes in the datasets, and to make the dataset size suitable for the parameterization method, given the available computational capacity).

\section{Appendix B. Selection of a suitable $\rho$-value for outlier compensation}

To identify a suitable value for the $\rho$ parameter in Eq. (5), handling the outlier compensation part of the likelihood calculations, ten full PSO cycles (250 iterations with 1000 Monte Carlo simulations in each) were performed on the most complex model variant (model variant BWGL, 10 free parameters), with different $\rho$-values. Since only a small part of the data can be assumed to be outliers, the $\rho$-values were sampled more densely closer to $\rho=1$. Fig. B1 shows the obtained values of the accumulator noise variance parameter $\sigma^{2}$ across these samples of $\rho$, each sample color scaled according to the corresponding total likelihood of the parameterized model. It can be observed that $\rho$-values above 0.8 and below 1 all generate noise values in the same region, all with a fairly high log-likelihood. For the remainder of the analysis, the $\rho$-value corresponding to the lowest $\sigma^{2}$ and highest loglikelihood was chosen, i.e. $\rho=0.9$.

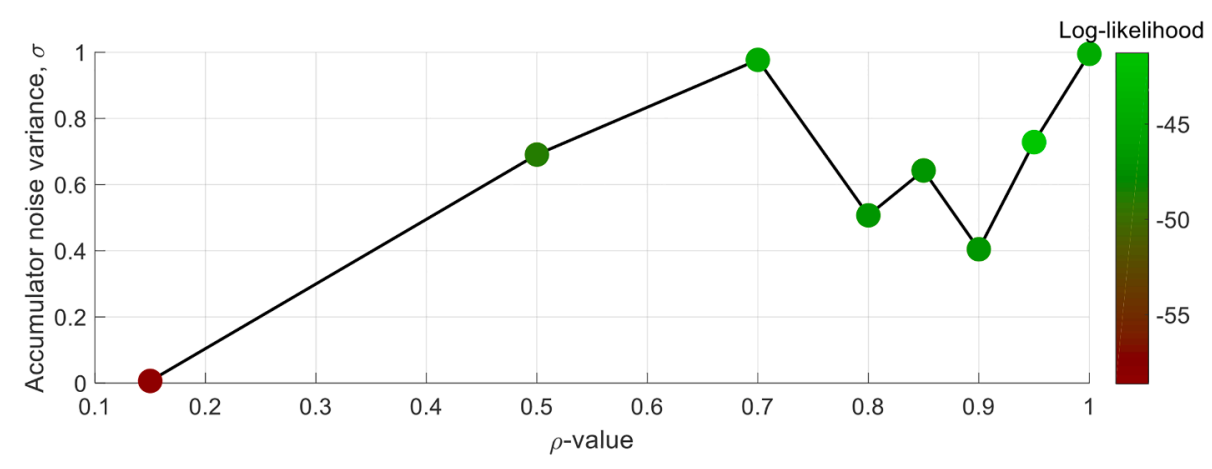

Fig. B1. Optimal values of the accumulator noise variance parameter $\sigma^{2}$ as a function of $\rho$-value. The corresponding log-likelihood value for the optimal parameter set is illustrated by the marker color, where dark red represents a low log-likelihood value and light green represents a high log-likelihood value. The analysis was made on model variant BWGL (10 free parameters). (For interpretation of the references to color in this figure legend, the reader is referred to the web version of this article.)

\section{Appendix C. Convergence of parameter values}

Since PSO in general does not guarantee convergence in a fixed number of iterations, the parameter value convergence was analyzed after each full PSO cycle. Convergence was assumed to be reached when all except a few particles agreed on a specific parameter value. That is, when the distribution of the Monte Carlo simulations peaked around the same value for almost all particles towards the final iterations in the PSO cycle. Since a few particles were still allowed to peak at other values, the convergence was analyzed by calculating the median absolute deviation (MAD) (see e.g. Leys, Ley, Klein, Bernard, \& Licata (2013) for each parameter. MAD is a measure of data variability that is robust to outliers and should be close to 0 for the model to have converged. Eventually, it was found that the PSO algorithm reached convergence in the parameter fitting of all model variants. See Fig. C1 for an example of MAD and optimal parameter value as a function of PSO iterations for all parameters in model BWL $\mathrm{L}_{\mathrm{rc}}$, parameterized on dataset $13 \mathrm{c}+39 \mathrm{nc}$. For illustrational purposes, Fig. C2 shows the corresponding histogram of values of the parameter for input weight during off road glance ( $w$ ) for each separate particle, for the last 75 iterations in the PSO cycle (out of 750). It can be observed that all particle histograms agree on the same value, but that, for example, particles 2, 6 and 16 have a slightly wider spread of values compared to the other particles. 

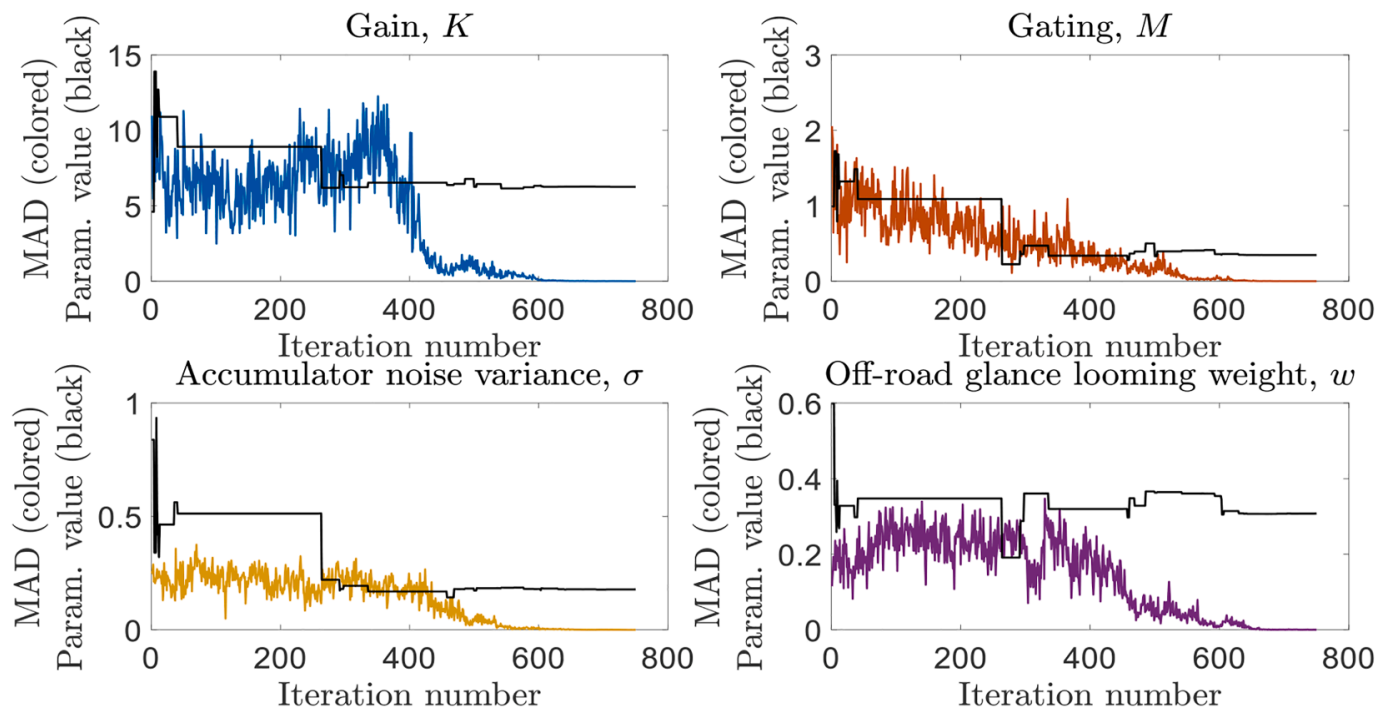

Fig. C1. Median absolute deviations (colored) and optimal parameter values (black) as a function of PSO iteration for the parameters in model BWL $\mathrm{rc}$

Off-road glance looming weight, $w$
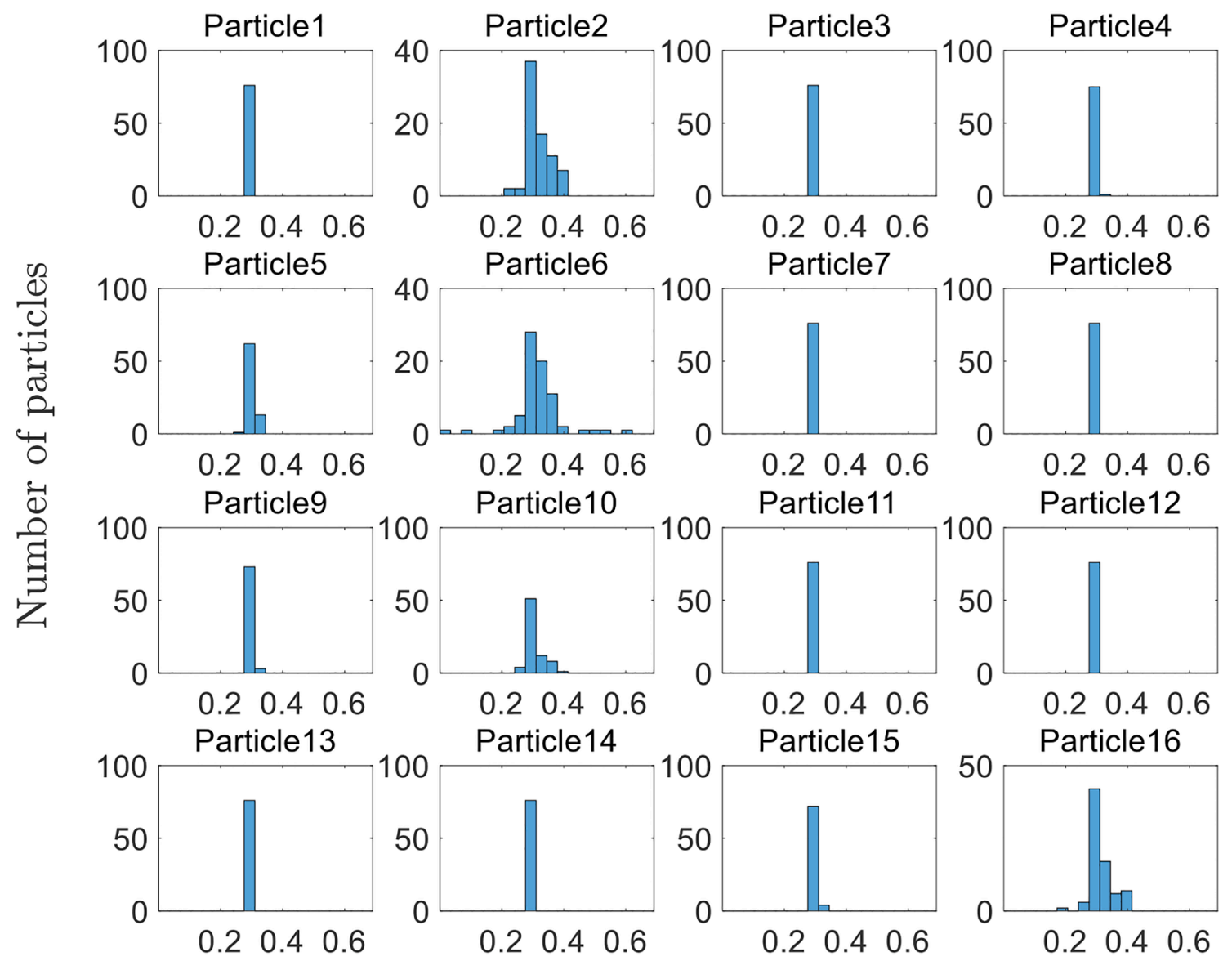

\section{Parameter value}

Fig. C2. Histograms, per particle, of the off-road glance looming weight parameter $(w)$ values for the last 75 iterations in the PSO cycle. 


\section{References}

Awad, W.H., Janson, B.N., 1998. Prediction models for truck accidents at freeway ramps in Washington State using regression and artificial intelligence techniques. Transp. Res. Rec. 1635 (1), 30-36. https://doi.org/10.3141/1635-04.

Bianchi Piccinini, G., Lehtonen, E., Forcolin, F., Engström, J., Albers, D., Markkula, G., Lodin, J., Sandin, J., 2020. How Do Drivers Respond to Silent Automation Failures? Driving Simulator Study and Comparison of Computational Driver Braking Models. Hum. Factors J. Hum. Factors Ergon. Soc. 62 (7), 1212-1229. https://doi.org/ 10.1177/0018720819875347.

Bärgman, J., Werneke, J., Boda, C.-N., Engström, J., Smith, K., 2013. Using Manual Measurements on Event Recorder Video and Image Processing Algorithms to Extrac Optical Parameters and Range, in: Proceedings of the 7th International Driving Symposium on Human Factors in Driver Assessment, Training, and Vehicle Design : Driving Assessment 2013. Bolton Landing, New York, pp. 177-183. doi:10.17077/ drivingassessment.1485.

Bärgman, J., Boda, C.N., Dozza, M., 2017. Counterfactual simulations applied to SHRP2 crashes: The effect of driver behavior models on safety benefit estimations of intelligent safety systems. Accid. Anal. Prev. 102, 165-180. https://doi.org/ 10.1016/j.aap.2017.03.003.

Burns, P.C., Andersson, H., Ekfjorden, A., 2000. Placing Visual Displays in Vehicles Where should they go ? International Conference on Traffic and Transportation Psychology.

Carsten, O., Kircher, K., Jamson, S., 2013. Vehicle-based studies of driving in the real world: The hard truth? Accid. Anal. Prev. 58, 162-174. https://doi.org/10.1016/j. aap.2013.06.006.

Crapse, T., Sommer, M., 2008. Corollary discharge circuits in the primate brain. Curr. Opinio Neurobiol. 18, 552-557. https://doi.org/10.1038/jid.2014.371.

Dingus, T.A., Guo, F., Lee, S., Antin, J.F., Perez, M., Buchanan-King, M., Hankey, J., 2016. Driver crash risk factors and prevalence evaluation using naturalistic driving data. Proc. Natl. Acad. Sci. 113 (10), 2636-2641. https://doi.org/10.1073/ pnas.1513271113.

Driver Focus-Telematics Working Group, 2006. Statement of Principles, Criteria and Verification Procedures on Driver Interactions with Advanced In- Vehicle Information and Communication Systems, Alliance of Automobile Manufacturers.

Engelbrecht, A.P., 2007. Computational Intelligence: An Introduction, 2nd ed. John Wiley \& Sons Ltd.

Engström, J., Bärgman, J., Nilsson, D., Seppelt, B., Markkula, G., Piccinini, G.B., Victor, T., 2018. Great expectations: a predictive processing account of automobile driving. Theor. Issues Ergon. Sci. 19 (2), 156-194. https://doi.org/10.1080/ 1463922X.2017.1306148.

Fajen, B.R., 2005. Calibration, information, and control strategies for braking to avoid a collision. J. Exp. Psychol. Hum. Percept. Perform. 31 (3), 480-501. https://doi.org/ 10.1037/0096-1523.31.3.480

Flach, J.M., Smith, M.R.H., Stanard, T., Dittman, S.M., 2004. Chapter 5. Collisions: Getting them under control. Adv. Psychol. 135, 67-91.

Giszter, S.F., 2015. MOTOR PRIMITIVES - New Data and Future Questions Simon. Curr. Opin. Neurobiol. 33, 156-165. https://doi.org/10.1016/j.physbeh.2017.03.040.

Gold, J.I., Shadlen, M.N., 2007. The Neural Basis of Decision Making. Annu. Rev. Neurosci. 30 (1), 535-574. https://doi.org/10.1146/annurev. neuro.29.051605.113038.

Green, M., 2000. "How long does it take to stop?" Methodological analysis of driver perception-brake times. Transp. Hum. Factors 2 (3), 195-216.

Guo, F., Klauer, S.G., Hankey, J.M., Dingus, T.A., 2010. Near crashes as crash surrogate for naturalistic Driving Studies. Transp. Res. Rec. 2147 (1), 66-74. https://doi.org/ 10.3141/2147-09.

Horrey, W.J., Wickens, C.D., 2007. In-Vehicle Glance Duration : Distributions, Tails, and Model of Crash Risk. Transp. Res. Board J. Transp. Res. Board 2018 (1), 22-28. https://doi.org/10.3141/2018-04.

Hurvich, C.M., Tsai, C.L., 1989. Regression and time series model selection in small samples. Biometrika 76 (2), 297-307. https://doi.org/10.1093/biomet/76.2.297.

Japan Automobile Manufacturers Association Inc., 2004. Guideline for In-vehicle Display Systems - Version 3.0.

Kiefer, R.J., Leblanc, D.J., Flannagan, C.A., 2005. Developing an inverse time-to-collision crash alert timing approach based on drivers' last-second braking and steering judgments. Accid. Anal. Prev. 37 (2), 295-303. https://doi.org/10.1016/j. aap.2004.09.003.

Kim, N.G., 2013. The Effect of Retinal Eccentricity on Perceiving Collision Impacts. Ecol. Psychol. 25 (4), 327-356. https://doi.org/10.1080/10407413.2013.839855.

Klauer, S.G., Guo, F., Simons-Morton, B.G., Ouimet, M.C., Lee, S.E., Dingus, T.A., 2014 Distracted driving and risk of road crashes among novice and experienced drivers. N. Engl. J. Med. 370 (1), 54-59. https://doi.org/10.1056/NEJMsa1204142.

Kusano, K.D., Gabler, H.C., 2012. Safety benefits of forward collision warning, brake assist, and autonomous braking systems in rear-end collisions. IEEE Trans. Intell. Transp. Syst. 13 (4), 1546-1555. https://doi.org/10.1109/TITS.2012.2191542.

Lamble, D., Laakso, M., Summala, H., 1999. Detection thresholds in car following situations and peripheral vision: implications for positioning of visually demanding in-car displays. Ergonomics 42 (6), 807-815. https://doi.org/10.1080/ 001401399185306.

Land, M., Horwood, J., 1995. Which parts of the road guide steering? Nature 377 (6547), 339-340. https://doi.org/10.1038/377339a0.

Lappi, O., Rinkkala, P., Pekkanen, J., 2017. Systematic Observation of an Expert Driver's Gaze Strategy_An On-Road Case Study. Front. Psychol. 8 https://doi.org/10.3389/ fpsyg. 2017.00620.

Lee, D.N., 1976. A Theory of Visual Control of Braking Based on Information about Timeto-Collision. Perception 5 (4), 437-459. https://doi.org/10.1068/p050437.
Lever, J., Krzywinski, M., Altman, N., 2016. Points of Significance: Model selection and overfitting. Nat. Methods 13 (9), 703-704. https://doi.org/10.1038/nmeth.3968.

Leys, C., Ley, C., Klein, O., Bernard, P., Licata, L., 2013. Detecting outliers: Do not use standard deviation around the mean, use absolute deviation around the median. J. Exp. Soc. Psychol.

Li, F.X., Laurent, M., 2001. Dodging a Ball Approaching on a Collision Path: Effects of Eccentricity and Velocity. Ecol. Psychol. 13 (1), 31-47. https://doi.org/10.1207/ S15326969ECO1301_2.

Markkula, G., 2014. Modeling driver control behavior in both routine and near-accident driving. Proc. Hum. Factors Ergon. Soc. 2014-Janua, 879-883. doi: 10.1177/ 1541931214581185.

Markkula, G., Benderius, O., Wolff, K., Wahde, M., 2012. A review of near-collision driver behavior models. Hum. Factors 54 (6), 1117-1143. https://doi.org/10.1177/ 0018720812448474.

Markkula, G., Boer, E., Romano, R., Merat, N., 2018. Sustained sensorimotor control as intermittent decisions about prediction errors: computational framework and application to ground vehicle steering. Biol. Cybern. 112 (3), 181-207. https://doi. org/10.1007/s00422-017-0743-9.

Markkula, G., Engström, J., Lodin, J., Bärgman, J., Victor, T., 2016. A farewell to brake reaction times? Kinematics-dependent brake response in naturalistic rear-end emergencies. Accid. Anal. Prev. 95, 209-226. https://doi.org/10.1016/j. aap.2016.07.007.

Morando, A., Victor, T., Dozza, M., 2016. Drivers anticipate lead-vehicle conflicts during automated longitudinal control: Sensory cues capture driver attention and promote appropriate and timely responses. Accid. Anal. Prev. 97, 206-219. https://doi.org/ 10.1016/j.aap.2016.08.025.

Murray, J.D., Bernacchia, A., Freedman, D.J., Romo, R., Wallis, J.D., Cai, X., PadoaSchioppa, C., Pasternak, T., Seo, H., Lee, D., Wang, X.-J., 2014. A hierarchy of intrinsic timescales across primate cortex. Nat. Neurosci. 17 (12), 1661-1663. https://doi.org/10.1038/nn.3862.

Najm, W.G., Smith, J.D., Yanagisawa, M., 2007. Pre-Crash Scenario Typology for Crash Avoidance Research. Dot Hs 810767 April, 128.

National Highway Traffic Safety Administration, 2012. Young Drivers Report the Highest Level of Phone Involvement in Crash or Near-Crash Incidences (DOT HS 811 611).

National Highway Traffic Safety Administration, 2016. Visual-Manual NHTSA Driver Distraction Guidelines for Portable and Aftermarket Devices June.

National Highway Traffic Safety Administration, 2020. Research Note: Distracted Driving 2018 (DOT HS 812 926).

Nunes, L.F., Gurney, K., 2016. Multi-alternative decision-making with non-stationary inputs. R. Soc. open sci. 3 (8), 160376. https://doi.org/10.1098/rsos.160376.

Page, Y., Fahrenkrog, F., Fiorentino, A., Gwehenberger, J., Helmer, T., Lindman, M., Op den Camp, O., van Rooij, L., Puch, S., Fränzle, M., Sander, U., Wimmer, P., 2015. A Comprehensive and Harmonized Method for Assessing the Effectiveness of Advance Driver Assistance Systems by Virtual Simulation. 24th Int. Tech. Conf. Enhanc. Saf. Veh. June.

Plöchl, M., Edelmann, J., 2007. Driver models in automobile dynamics application. User Modeling and User-Adapted Interaction 45 (7-8), 699-741. https://doi.org/ 10.1080/00423110701432482.

Ratcliff, R., Van Dongen, H.P.A., 2011. Diffusion model for one-choice reaction-time tasks and the cognitive effects of sleep deprivation. Proc. Natl. Acad. Sci. 108 (27), 11285-11290. https://doi.org/10.1073/pnas.1100483108.

Robertshaw, K.D., Wilkie, R.M., 2008. Does gaze influence steering around a bend? J. Vis. 8 (4), 1-13. https://doi.org/10.1167/8.4.18.

Shi, Y., Eberhart, R.C., 1998. Parameter selection in particle swarm optimization. In: International Conference on Evolutionary Programming. Springer, Berlin, Heidelberg, pp. 591-600. https://doi.org/10.1007/BFb0040810.

Stoffregen, T., Riccio, G., 1990. Responses to Optical Looming in the Retinal Center and Periphery. Ecol. Psychol. 2 (3), 251-274. https://doi.org/10.1207/ s15326969eco0203_3.

Sugiura, N., 1978. Further Analysis of the Data by Anaike' S Information Criterion and the Finite Corrections. Commun. Stat. - Theory Methods 7 (1), 13-26. https://doi. org/10.1080/03610927808827599.

Summala, H., Lamble, D., Laakso, M., 1998. Driving experience and perception of the lead car's braking when looking at in-car targets. Accid. Anal. Prev. 30 (4), 401-407.

Summala, H., Nieminen, T., Punto, M., 1996. Maintaining lane position with peripheral vision during in-vehicle tasks. Hum. Factors 38 (3), 442-451. https://doi.org/ 10.1518/001872096778701944.

Svärd, M., Bärgman, J., Victor, T., 2020. Detection and response to critical lead vehicle deceleration events with peripheral vision: Glance reaction times are independent of visual eccentricity. Submitt. Publ.

Svärd, M., Markkula, G., Engström, J., Granum, F., Bärgman, J., 2017. A quantitative driver model of pre-crash brake onset and control, in: Proceedings of the Human Factors and Ergonomics Society. doi:10.1177/1541931213601565.

Tarko, A.P., 2018. Surrogate measures of safety. Transp. Sustain. 11, 383-405. https:// doi.org/10.1108/S2044-994120180000011019.

The Commision of European Communities, 2008. Commision recommendation of 26 May 2008 on safe and efficient in-vehicle information and communication systems: update of the European Statement of Principles on human-machine interface, Official Journal of the European Union.

Transportation Research Board of the National Academy of Sciences, 2013. The 2nd Strategic Highway Research Program Naturalistic Driving Study Dataset. Available from SHRP 2 NDS InSight Data Dissem. web site.

Usher, M., McClelland, J.L., 2001. The time course of perceptual choice: The leaky, competing accumulator model. Psychol. Rev. 108 (3), 550-592. https://doi.org/ 10.1037/0033-295X.108.3.550. 
Van Den Bergh, F., Engelbrecht, A.P., 2006. A study of particle swarm optimization particle trajectories. Inf. Sci. (Ny) 176 (8), 937-971. https://doi.org/10.1016/j. ins.2005.02.003.

Victor, T., Dozza, M., Bärgman, J., Boda, C.N., Engström, J., Flannagan, C., Lee, J.D., Markkula, G., 2014. Analysis of Naturalistic Driving Study Data: Safer Glances, Driver Inattention, and Crash Risk, Analysis of Naturalistic Driving Study Data: Safer Glances, Driver Inattention, and Crash Risk. doi:10.17226/22297.

Wahde, M., 2008. In: Biologically inspired optimization methods: an introduction. WIT Press. https://doi.org/10.5860/choice.46-3899.

Wolfe, B., Dobres, J., Rosenholtz, R., Reimer, B., 2017. More than the Useful Field: Considering peripheral vision in driving. Appl. Ergon. 65, 316-325. https://doi.org/ 10.1016/j.apergo.2017.07.009.
Wolfe, B., Sawyer, B.D., Kosovicheva, A., Reimer, B., Rosenholtz, R., 2019. Detection of brake lights while distracted: Separating peripheral vision from cognitive load. Attention, Perception, Psychophys. 81 (8), 2798-2813. https://doi.org/10.3758/ s13414-019-01795-4.

Zhang, Y., Wang, S., Ji, G., 2015. A Comprehensive Survey on Particle Swarm Optimization Algorithm and Its Applications. Math. Probl. Eng. 2015, 1-38. https:// doi.org/10.1155/2015/931256.

Zheng, L., Ismail, K., Meng, X., 2014. Traffic conflict techniques for road safety analysis: Open questions and some insights. Can. J. Civ. Eng. 41 (7), 633-641. https://doi. org/10.1139/cjce-2013-0558. 